\title{
The rheology and microstructure of acicular precipitated calcium carbonate colloidal suspensions through the shear thickening transition
}

\author{
Ronald G. Egres and Norman J. Wagner ${ }^{\text {a) }}$ \\ Department of Chemical Engineering and Center for Molecular and Engineering \\ Thermodynamics, University of Delaware, Newark, Delaware 19716
}

(Received 11 November 2004; final revision received 21 February 2005)

\begin{abstract}
Synopsis
The shear rheology and shear-induced microstructure of poly(ethylene glycol) (PEG)-based suspensions of acicular precipitated calcium carbonate (PCC) particles of varying particle aspect ratio (nominal $L / D \sim 2,4,7$ ) are reported. These anisotropic particle suspensions demonstrate both continuous and discontinuous reversible shear thickening with increasing applied shear rate or stress similar to that observed for suspensions of spherical colloidal particles. The critical volume fraction for the onset of discontinuous shear thickening decreases as the average particle aspect ratio is increased. However, the critical stress for shear thickening is found to be independent of particle anisotropy and volume fraction. Rather, it can be predicted based on the minor axis diameter of the particles and is found to agree with values for near hard-sphere suspensions. Small angle neutron scattering during shear flow (Rheo-SANS) demonstrates that long-axis particle alignment with the flow direction is maintained throughout the range of shear stresses investigated, including the shear thickening regimes for both continuously and discontinuously shear thickening PCC/PEG suspensions. Rheo-SANS and transient rheological experiments indicate that this reversible shear thickening is a consequence of lubrication hydrodynamic interactions and the formation of transient hydroclusters of flow-aligned particles. (C) 2005 The Society of Rheology. [DOI: 10.1122/1.1895800]
\end{abstract}

\section{INTRODUCTION}

Suspensions containing anisotropic particles, such as clays, pigments, and inorganic mineral crystals have significant commercial importance in several industrial applications. The manufacture of paper products often employs suspensions of clays such as kaolin as well as precipitated calcium carbonate (PCC) to improve paper opacity and gloss factor [Lee and Reder (1972); Beazley (1980)]. In industrial paper coating processes, it is advantageous to apply anisotropic particle coating suspensions with high solids loadings to reduce the drying time required for solvent removal. Processing speeds and coating quality are often limited by the suspension rheology. Qualitatively, concentrated suspensions containing anisotropic particles demonstrate similar rheological responses to shear flow as observed in concentrated spherical (isotropic) particle suspensions, such as reversible shear thinning and shear thickening, yielding behavior, thixotropy, etc. [Lee and Reder (1972); Beazley (1980)]. Rheological and microstructural

${ }^{a}$ Author to whom correspondence should be addressed; electronic mail: wagner@che.udel.edu 
differences observed between anisotropic and spherical particle suspensions are often attributed to excluded volume effects; where increasing particle anisotropy results in geometrically hindered particle motion at particle loadings significantly less than the maximum packing fraction [Jogun and Zukoski (1996)]. Of particular concern to coating and transport processes is that shear thickening in anisotropic particle suspensions can damage process equipment and lead to poor coating quality [Beazley (1980)].

The reversible shear thickening transition in suspensions of spherical particles has been studied extensively in the literature. A characteristic of the shear rheology of spherical silica and plastisol suspensions is that shear thinning is observed with increasing shear rate, which may be followed by shear thickening at higher shear rates [Hoffman (1972); Laun (1984); Maranzano and Wagner (2001a)]. It is believed that the increase in viscosity observed during reversible shear thickening in concentrated colloidal suspensions is the result of shear induced microstructural changes that occur within the fluid. Early investigations attribute the change in rheological response from shear thinning to shear thickening behavior to an order-disorder transition within the concentrated suspension [Hoffman $(1972,1974)]$. The disruption of a flow-ordered structure was thought to result in increased particle interactions, leading to increases in suspension viscosity [Hoffman (1974); Boersma et al. (1992); Laun et al. (1992)]. Simulations [Brady and Bossis (1988); Catherall et al. (2000)] and experiments [D'Haene et al. (1992); Bender and Wagner (1995); Kaffashi et al. (1997); O'Brien and Mackay (2000); Maranzano and Wagner (2002); Newstein et al. (1999)] have shown that reversible shear thickening results from the formation of hydroclusters, or transient stress bearing particle aggregates that form as a consequence of short range hydrodynamic lubrication forces overcoming the interparticle repulsive forces during flow. Percolation of these hydroclusters with increasing shear results in the formation of larger aggregates that can jam the flow, leading to discontinuous shear thickening behavior. Note that an underlying orderdisorder transition is neither necessary nor sufficient to trigger shear thickening [Laun et al. (1992); Bender and Wagner (1995)], although order-disorder transitions may accompany the shear thickening transition [Hoffman (1972); Laun et al. (1992)]. Finally, scaling models for the prediction of critical stress based on particle size and particle interaction potential have been developed and verified through experimentation [Maranzano (2001); Maranzano and Wagner (2001a, 2001b); Gopalakrishnan and Zukoski (2004)].

Although shear thickening in anisotropic particle colloidal suspensions, such as clay and pigment suspensions, is observed in industrial practice, little work has been done to develop predictive models or elucidate the mechanism for this shear thickening response. For coarse (noncolloidal) suspensions of glass rods and plates, Clarke (1967) demonstrated that particle shape can dramatically affect shear thickening behavior. Several reports demonstrate shear thickening behavior in aqueous clay suspensions [Beazley (1980); Alince and Lepoutre (1983); Lee and Reder (1972); O'Brien and Mackay (2002)]. From rheological investigations of colloidal kaolin clay suspensions having mean particle length to thickness ratios of 5 and 50, Beazley (1980) observed that increasing the aspect ratio of disk-shaped clay particles resulted in shear thickening behavior at lower particle loadings. Beazley (1980) argues that high aspect ratio clay suspensions demonstrate shear thickening at low particle volume fractions because of poor particle packing efficiency in the unaligned state. Elongational thickening behavior in kaolin clay suspensions has also been explored through the experimental work of O'Brien and Mackay (2002).

Shear thickening has also been observed in colloidal suspensions of rod-like anisotropic particles. Rheological investigations of suspensions of ceramic materials by Berg- 
strom (1996) demonstrated that aqueous suspensions of rod-shaped $(L / D \sim 10)$ silicon carbide whiskers exhibited discontinuous shear thickening at volume fractions as low as $\phi=0.172$.

Both Beazley (1980) and Bergstrom (1996) attribute the shear thinning observed in anisotropic particle suspensions to particle alignment with flow, while the observed shear thickening is attributed to increased collisions between particles resulting from misalignment at higher shear rates. Beazley (1980) suggests that particle rotation during flow may result in particles interlocking and jamming in disorganized clusters. This type of instability is thought to increase with increasing particle anisotropy, as rods and disks with higher aspect ratio should "entangle" to form aggregates of randomly oriented anisotropic particles more readily at lower particle loadings, ultimately resulting in the observed shear thickening behavior. Note that Philipse (1996) provides independent evidence for the effect of aspect ratio on particle packing in the unaligned state, reporting sedimentation studies conducted on several rod-like colloidal suspensions that demonstrate a reduction in the random packing density with increasing particle anisotropy. Bergstrom (1998) also postulates that shear thickening in rod-shaped suspensions is a result of the breakup of long-range order achieved during shear thinning and possibly the formation of hydrodynamic clusters, which may be a consequence of a similar misalignment and jamming mechanism. However, neither Beazley (1980) nor Bergstrom (1998) provide direct microstructural evidence in support of the hypothesis that shear thickening in anisotropic particle suspensions is a consequence of particle misalignment at higher shear rates.

Flow-induced microstructure in anisotropic particle suspensions subject to shearing flows has been measured using various techniques. Simultaneous rheological and small angle x-ray scattering studies of shear thinning suspensions of mineral particles have been performed by Camerel et al. (2003). Light and small angle neutron scattering have been used to examine the effects of particle volume fraction on the microstructure of disk-shaped clay suspensions during shear [Ramsay and Linder (1993)]. In the work of Clarke et al. (1996), neutron diffraction experiments conducted on flowing kaolinite clay particle suspensions generated anisotropic scattering patterns, the result of scattering from crystal planes within plate-like kaolinite crystals which flow align during shear flow. Several researchers have determined anisotropic particle alignment in sheared suspensions through measurement of conductivity after flow cessation [Jogun and Zukoski (1996); Dellimore and Gosling (1973); Okubo (1987)]. However, to date there have been no measurements of the shear-induced microstructure of flowing anisotropic particle suspensions in the shear thickening regime, and hence, no quantitative evidence to support the hypothesis of flow-disordering being responsible for shear thickening in these systems.

To address the issues surrounding the mechanisms of reversible shear thickening in anisotropic particle suspensions, we investigated the rheology and flow-induced microstructure of model suspensions of acicular PCC particles of similar nominal small axis diameter but different long axial dimensions. For this investigation, the PCC particles were resuspended in $200 M_{w}$ poly(ethylene glycol) $\left(\rho=1.121 \mathrm{~g} \mathrm{~cm}^{-3}, \eta=0.049 \mathrm{~Pa} \mathrm{~s}\right.$ at $25^{\circ} \mathrm{C}$ ) to yield stable suspensions for use in rheological and small angle neutron scattering (SANS) investigations. The experimental results of this work provide four fundamental observations: (a) systematic increase in the average particle aspect ratio of PCC/ poly(ethylene glycol) (PEG) suspensions results in a reduction in the particle loading required to achieve discontinuous shear thickening behavior, (b) particle aspect ratio is observed to have negligible influence on the critical stress values associated with the shear thickening transition, (c) the observed, rapid response to transient step shear and 
TABLE I. Particle properties of PCC/PEG suspensions, $D_{r 0}$ is the bare particle rotational diffusion coefficient in PEG calculated from the particle geometry.

\begin{tabular}{lccccc}
\hline \hline Particle & $\begin{array}{c}\text { Length, } \\
L(\mathrm{~nm})\end{array}$ & $\begin{array}{c}\text { Diameter, } \\
D(\mathrm{~nm})\end{array}$ & $\begin{array}{c}\text { Aspect ratio } \\
L / D\end{array}$ & $\begin{array}{c}D_{r 0} \\
\left(\mathrm{~s}^{-1}\right)\end{array}$ & $\begin{array}{c}\text { Density } \\
\left(\mathrm{g} / \mathrm{cm}^{3}\right)\end{array}$ \\
\hline PCC(2) & $567 \pm 217$ & $328 \pm 103$ & $1.72 \pm 0.34$ & $0.330 \pm 0.091$ & $2.68 \pm 0.01$ \\
PCC(4) & $1004 \pm 465$ & $233 \pm 71$ & $4.30 \pm 1.45$ & $0.134 \pm 0.111$ & $2.83 \pm 0.01$ \\
PCC(7) & $1323 \pm 667$ & $201 \pm 77$ & $6.68 \pm 2.56$ & $0.074 \pm 0.076$ & $2.86 \pm 0.01$ \\
\hline \hline
\end{tabular}

flow reversal experiments conducted during shear thinning and shear thickening contradicts expectations of the proposed entanglement mechanism for shear thickening, and (d) flow-small angle neutron scattering (flow-SANS) and Rheo-SANS investigations of the PCC/PEG suspensions at applied stresses beyond the shear thickening transition demonstrate that long axis particle alignment with flow direction is largely maintained throughout the shear thickening regime for both continuous and discontinuous shear thickening suspensions. These observations suggest that the mechanism responsible for shear thickening in anisotropic particle suspensions is analogous to the transient hydrocluster mechanism observed in spherical particle suspensions. As a consequence, we demonstrate a quantitative prediction for the critical stress at the onset of shear thickening in suspensions of acicular or prolate ellipsoidal particles based on previous work for near hardsphere suspensions.

\section{MATERIALS AND METHODS}

\section{A. Suspension preparation and characterization}

Aqueous acicular PCC suspensions (Specialty Minerals, Inc., Bethlehem, PA) with three different particle aspect ratios were used to generate the suspensions for this investigation. These suspensions are commercially available under the trade names Jay Albaglos S, Opacarb A40, and MD1074, and are reported to have average particle aspect ratios $(L / D)$ of approximately 2,4 , and 7 , respectively. The densities for each type of PCC particle (dispersed in de-ionized water) were measured via solution densimetry performed using a density meter (Anton Paar, model DMA 48) and are reported in Table I. Particle densities were determined from a series of measurements on dilute suspensions under the assumption of ideal mixing [Maranzano (2001); Maranzano and Wagner (2001a)].

The PCC particles were resuspended in $200 M_{w}$ reagent grade poly(ethylene glycol) (Acros Chemicals) through repeated centrifugation and solvent exchange. Three centrifugation, solvent exchange, and resuspension steps were performed to prepare concentrated PEG/PCC suspensions of each aspect ratio. Centrifugation of the commercial, aqueous suspensions was performed using a desktop centrifuge, (International Equipment Co., model CL Centrifuge) operating at $1750 \mathrm{~g}$ for $2 \mathrm{~h}$. Resuspensions were performed through the addition of PEG equal in volume to that of the resulting sediment, followed by agitation using a rolling jar mixer for redispersion. Centrifugation for particle separation from PEG was conducted at 16,000 g using a high-speed centrifuge (DuPont Instruments, RC-5) for $2 \mathrm{~h}$. The final sediment from each of the PCC materials was redispersed in PEG to produce concentrated suspensions having particle volume fractions near $\phi$ $=0.50$, and an estimated final water concentration in the suspending medium below 2 wt \%. All dilute PCC/PEG samples generated at the particle volume concentrations reported in this investigation were produced assuming ideal mixing based on the measured 

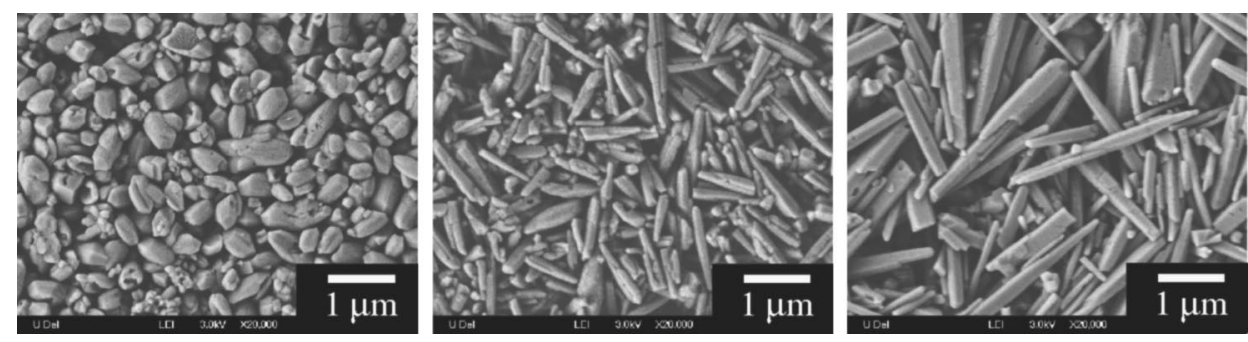

FIG. 1. SEM images $(20,000 \times)$ of precipitated calcium carbonate particles from (a) PCC(2)/PEG, (b) PCC(4)/ PEG, and (c) PCC(7)/PEG suspensions.

densities of the particles and PEG. Following preparation, dilute samples were sonicated using an ultrasonic bath for $1 \mathrm{~h}$, followed by $24 \mathrm{~h}$ of mixing using the rolling jar mixer to facilitate dispersion of the particles. Particle concentration was verified through measurement of final suspension density using the solution density meter. These diluted PEG-based suspensions having mean PCC particle aspect ratios $(L / D)$ of roughly 2,4 , and 7 are herein referred to as PCC(2)/PEG, PCC(4)/PEG, and PCC(7)/PEG, respectively.

Figure 1 presents scanning electron microscopy (SEM) (JEOL JSM-7400F, JEOL Ltd.) micrographs taken at $20,000 \times$ magnification of the particles from each of the three PEG/PCC suspensions investigated. Table I presents the average particle length, $L$ (long axis dimension), diameter, $D$ (small axis dimension), and aspect ratio, $L / D$ based on transmission electron microscopy (TEM) (JEOL JEM-2000FX, JEOL Ltd.) measurements of 500 particles from each of the PCC/PEG suspensions using image analysis software (Scion Image, Scion Corporation). The particles in each of the suspensions are polydisperse in both particle shape and size. In the TEM image analysis it was assumed that the particles were uniaxial, although some evidence for slight biaxiality is evident in the SEM images.

\section{B. Rheology}

Prior to testing, all samples were agitated for $12 \mathrm{~h}$ using a vortex mixer (Maxi-Mix ${ }^{\circledR}$ III, Barnstead/Thermolyne Corp.) to ensure complete dispersion of the particles. Rheological measurements conducted using a controlled stress rheometer (SR 5000, Rheometric Scientific, Inc.) with a cone and plate geometry (0.02 rad, $25 \mathrm{~mm}$ diameter cone) were performed at $25 \pm 0.01{ }^{\circ} \mathrm{C}$ with peltier temperature control. The following preshear/stress ramp protocol was performed on each sample prior to conducting any of the experiments reported:

(1) following loading, a preshear stress ramp from 0.3 to $300 \mathrm{~Pa}$ was conducted to eliminate any effects of sample loading and

(2) following the preshear, a second stress ramp from 0.3 to $300 \mathrm{~Pa}$ in $300 \mathrm{~s}$ and back to $0.3 \mathrm{~Pa}$ in $300 \mathrm{~s}$ was performed to demonstrate the reversibility of any shear thickening observed in these suspensions.

Stress sweeps were performed on samples from $0.3 \mathrm{~Pa}$ to a maximum stress of 10,000 $\mathrm{Pa}$. For samples with lower particle volume fractions, this maximum stress value could not be achieved due to the rate limitations of the rheometer, so measurements were recorded to the maximum stress attainable. Ascending and descending stress sweeps were also performed from 0.3 to $1000 \mathrm{~Pa}$ and back to $0.3 \mathrm{~Pa}$, demonstrating minimal hysteresis 
such that the critical stress for shear thickening was observed to be independent of stress sweep direction. Stress sweep measurements made using parallel plate $(25 \mathrm{~mm})$ and Couette cell (17 mm DIN, $0.25 \mathrm{~mm}$ gap) geometries were performed to determine the effects of tool geometry on the measured rheology of these anisotropic particle colloidal systems. Apparent wall slip was characterized by performing stress sweeps on PCC/ PEG(7) suspensions exhibiting both continuous and discontinuous shear thickening using a parallel plate geometry at several gap heights [Yoshimura and Prud'homme (1988)]. Creep relaxation experiments at several applied stresses using this controlled stress instrument were performed to determine the necessary delay time for steady shear measurements, as well as to measure any recoverable compliance. Flow reversal experiments were conducted at several shear rates within the shear thinning regime, near the shear thickening transition, and within the shear thickening regime. Prior to and following the point of flow reversal, samples were sheared at the prescribed shear rate for $60 \mathrm{~s}$.

A controlled strain rheometer (ARES, TA Instruments, Inc.) equipped with a wave form and fast data sampling option (National Instruments DAQ-Pad 6020E, $100 \mathrm{kS} / \mathrm{s}$ sampling rate) was used to characterize transient rheological behavior for step rate experiments using a $25 \mathrm{~mm}, 0.02$ rad cone geometry. Prior to performing step rate experiments, a thixotropic loop test was performed as a preshear to a maximum shear rate of $300 \mathrm{~s}^{-1}$ in $300 \mathrm{~s}$, and back to $0 \mathrm{~s}^{-1}$ in $300 \mathrm{~s}$. Step rate experiments were conducted at a low shear rate within the shear thinning regime, followed by a step increase to a higher rate where the samples exhibited shear thickening behavior, and finally, back to the low shear rate.

A controlled stress rheometer (MDS500, Paar Physica) with a large Couette geometry (26.66 $\mathrm{mm}$ bob diameter, $40 \mathrm{~mm}$ gap length, $28.92 \mathrm{~mm}$ cup internal diameter) was used to measure the steady shear rheology of PCC/PEG samples prior to performing RheoSANS experiments using comparable instrumentation at the National Institute of Standards and Technology (NIST, Gaithersburg, MD) NG-7 beam line.

\section{SANS}

Static small angle neutron scattering experiments were performed on $\mathrm{PCC}(7) / \mathrm{PEG}$ suspension samples at various concentrations loaded into sandwich cells at the National Center for Neutron Research at NIST on a 30 m SANS instrument (NG-3) using thermal neutrons with a $6 \AA$ wavelength (14.7\% full width of half maximum) at a sample to detector distance of $12 \mathrm{~m}$.

Although static SANS cannot be used as a means to unambiguously measure the shape and polydispersity of these relatively large particles, flow-SANS experiments have proven to be an effective means of detecting flow alignment and even estimating particle dimensions for objects of comparable size [Walker and Wagner (1996); Kernick and Wagner (1999)]. For example, Kernick and Wagner (1999) characterized the threedimensional structure of flow-induced, ellipsoidal, immiscible polymer blend inclusions having dimensions on the order of $1 \mu \mathrm{m}$ using flow-SANS. Through combining the on-axis and tangential scattering results during shear flow, the full geometry of the ellipsoid and the tilt angle in the shear direction were resolved.

Flow-SANS experiments were conducted using a $30 \mathrm{~m}$ SANS instrument (NG-3, NIST). Measurements were taken on PCC(7)/PEG suspensions exhibiting continuous shear thickening behavior $(\phi=0.25$ and $\phi=0.29)$ using a quartz Couette shear cell $(10 \mathrm{~cm}$ DIN, $0.5 \mathrm{~mm}$ gap) driven by a stepper motor (no torque measurement capability). Scattering data at a sample to detector distance of $12 \mathrm{~m}$ resulting from the neutron beam directed both radially (on-axis) and tangentially (off-axis) through the sample during 


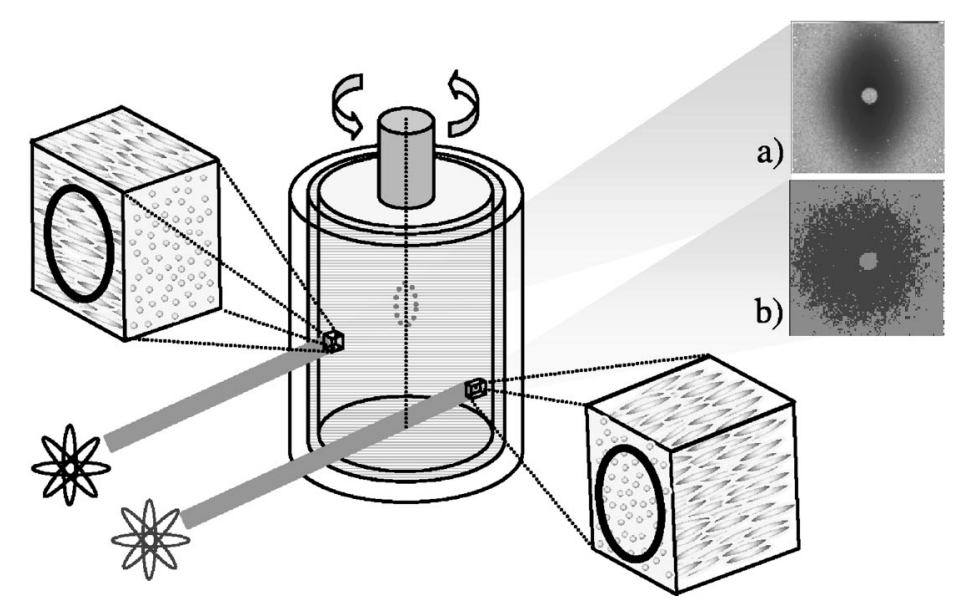

FIG. 2. Rheo-SANS shear cell configuration and the resulting on-axis (a) and off-axis (b) scattering patterns generated from the concentrated PCC (7)/PEG suspension $(\phi=0.35)$ during shear flow $(\tau=500 \mathrm{~Pa})$. Anisotropy observed in the on-axis pattern and the isotropic off-axis scattering pattern suggests long axis particle alignment with flow direction, as illustrated in the magnified sketches of particle alignment within the cell.

shear flow was obtained, providing information on structural changes that occur with increasing shear in the velocity-vorticity plane and the gradient-vorticity plane, respectively.

Additional Rheo-SANS experiments were conducted on a $30 \mathrm{~m}$ SANS instrument (NG-7, NIST) equipped with a rheometer (Paar Physica, model UDS 200) providing real time experimental torque measurement and controlled stress operation. These "RheoSANS" experiments were performed on $\mathrm{PCC}(7) / \mathrm{PEG}$ suspensions samples exhibiting continuous $(\phi=0.28)$ and discontinuous $(\phi=0.35)$ shear thickening behavior in a Couette type shear cell consisting of an quartz cup $(5 \mathrm{~cm}$ DIN) and a quartz bob $(4.8 \mathrm{~cm}$ outer diameter) at a sample to detector distance of $15 \mathrm{~m}$. Scattering patterns resulting from the samples during shear flow were obtained with the neutron beam directed in both the radial (on-axis) and tangential (off-axis) directions. A $1 \mathrm{~mm}$ slit was used to guide the beam through the gap in the Couette cell during tangential scattering measurements performed using this experimental arrangement.

Figure 2 illustrates the experimental scattering arrangement used for the Rheo-SANS and flow-SANS experiments, along with sketches of particle alignments corresponding to scattering patterns typically observed in this work. The resulting intensity versus scattering vector data from the experiments were reduced and put on an absolute scale using NIST standard software.

\section{RESULTS}

\section{A. Rheology of precipitated calcium carbonate suspensions}

Ascending and descending stress sweeps were performed on PCC/PEG suspensions using the stress controlled rheometer with cone and plate geometry, illustrating the reversible nature of the shear thickening observed in these systems. Figure 3(a) presents two sets of ascending and descending stress sweeps performed consecutively on $\mathrm{PCC}(7) /$ PEG suspensions exhibiting continuous $(\phi=0.28)$ and discontinuous $(\phi=0.35)$ shear thickening at applied stresses above approximately $10 \mathrm{~Pa}$. Experiments were performed two days after sample preparation and following $12 \mathrm{~h}$ of vortex mixing. The samples 

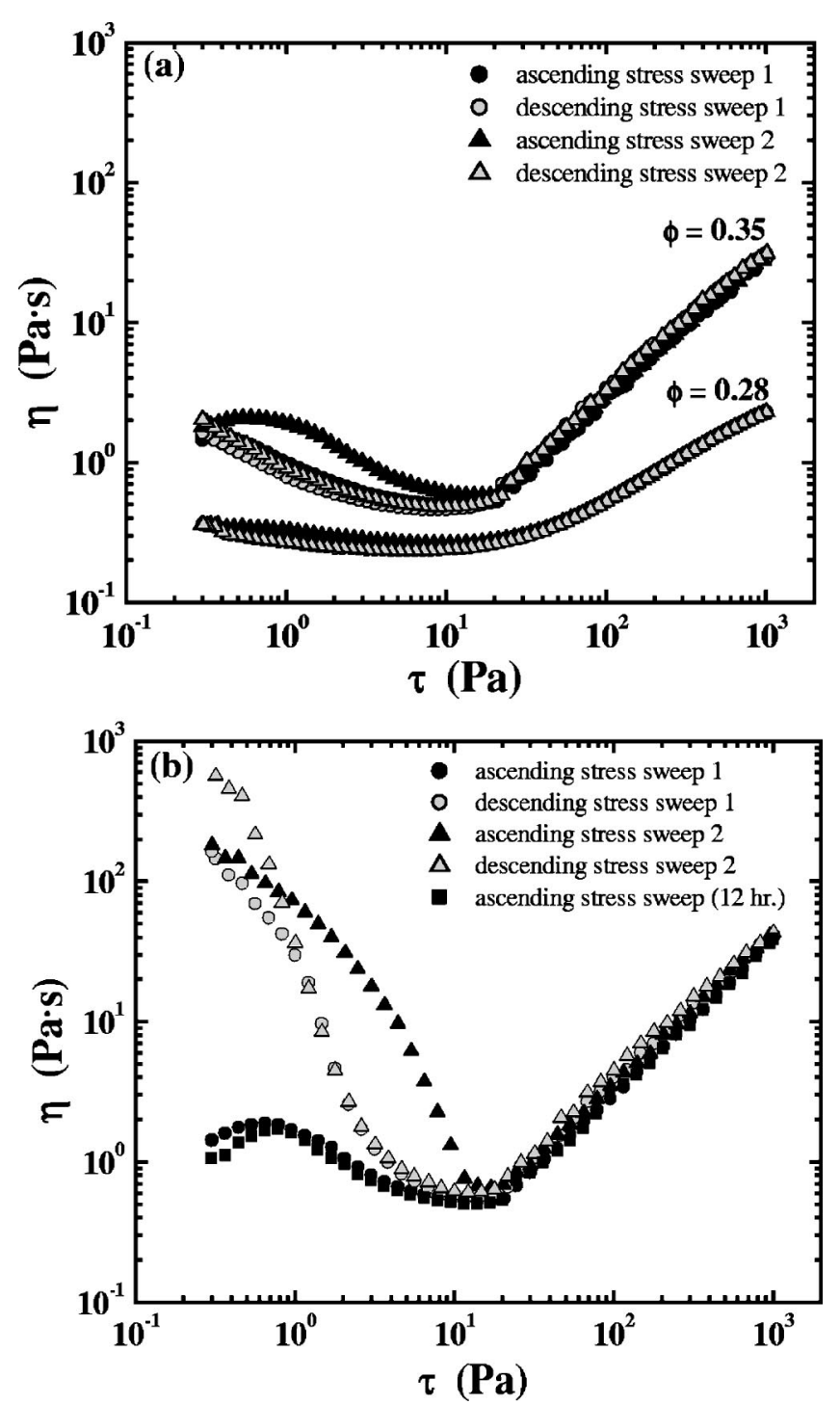

FIG. 3. Effect of sample aging on PCC(7)/PEG suspensions: (a) ascending and descending stress sweeps 2 days following sample preparation for PCC(7)/PEG suspensions at $\phi=0.28$ and $\phi=0.35$, (b) ascending and descending stress sweeps 40 days following sample preparation for the PCC(7)/PEG dispersion at $\phi=0.35$. Filled squares represent an ascending stress sweep performed $12 \mathrm{~h}$ after the initial series of ascending and descending stress sweeps.

were allowed to rest for $30 \mathrm{~s}$ between each successive stress sweep experiment. The increase in low shear viscosity observed during the second ascending stress sweep for the PCC(7)/PEG, $\phi=0.35$ suspension may suggest that extended exposure to high shear stresses in the shear thickening regime during the preceding ascending and descending stress sweeps may affect the sample's rest state. In Fig. 3(b), ascending and descending stress sweeps were performed on the PCC(7)/PEG, $\phi=0.35$ sample 40 days following sample preparation. After 40 days of standing, followed by $12 \mathrm{~h}$ of sediment redispersion via vortex mixing, the initial forward stress sweep is comparable to that obtained in the last forward sweep shown in Fig. 3(a). However, during the first descending stress sweep 
and subsequent ascending and descending stress sweeps, evidence of a yield stress is apparent. It is important to note that in all flow curves presented in Fig. 3, the viscosity just prior to the onset of shear thickening and the viscosity within the shear thickening regime were found to be reproducible independent of the sample's shear history or age.

The sensitivity of the low shear viscosity to shear history and sample age was most apparent in concentrated samples exhibiting discontinuous shear thickening behavior. One possible explanation for this behavior is that the high shear particle interactions associated with discontinuous shear thickening might facilitate the desorption of surface adsorbed species (such as residual adsorbed polymeric stabilizing agents present in the supplied aqueous PCC dispersions) from the surface of the particles. This might act to reduce interparticle stabilizing forces between particles and induce reversible aggregation within the sample which can subsequently be eliminated with increasing shear. The tendency for this desorption with prolonged exposure to shear thickening may conceivably increase with sample age. Interestingly, an ascending stress sweep (after repeating the preshear/stress sweep protocol) performed on this same 40-day-old PCC(7)/PEG sample allowed to rest $12 \mathrm{~h}$ following the original stress sweep sequence demonstrates comparable flow behavior to that of the initial ascending steady shear curve, exhibiting no evidence of permanent aggregation as a result of shear history [Fig. 3(b)]. This might suggest that after a sufficient period of rest following extensive shear thickening, the desorbed species are able to migrate from the bulk suspending medium and readsorb to the surface of the particles, resulting in improved dispersion of the particles.

The effects of tool geometry and gap dimension on the steady shear rheology of the PCC(7)/PEG suspensions are explored in Fig. 4. Figure 4(a) presents viscosity versus stress measurements of the PCC(7)/PEG system at $\phi=0.28$ and $\phi=0.35$ using cone and plate, parallel plate, and Couette geometries. Stress sweeps performed using the Couette and the cone and plate illustrate good agreement, demonstrating that the rheology of these suspensions of acicular particles are independent of tool geometry for shearing flows having a homogeneous strain field.

Variable gap experiments performed on PCC(7)/PEG suspensions using the $25 \mathrm{~mm}$ parallel plate geometry illustrate that gap height influences the measured rheology, which is a signature of wall slip [Yoshimura and Prud'homme (1988); Chow and Zukoski (1995); Maranzano (2001)]. To quantify the wall slip in the anisotropic PCC(7)/PEG suspensions, parallel plate rheology data taken at several gap heights is analyzed using the method reported by Yoshimura and Prud'homme (1988). Gap heights ranging from $20 \mu \mathrm{m}$ to $1 \mathrm{~mm}$ were investigated for PCC(7)/PEG suspensions at $\phi=0.28$ and $\phi=0.35$, as well as for a $5 \mathrm{~Pa}$ oil standard. Linear fits to the parallel plate data plotted as apparent (measured) shear rate $\left(\dot{\gamma}_{\text {apparent }}\right)$ versus reciprocal gap height at fixed plate edge stress $\left(\tau_{R}\right)$ values were used to characterize the apparent slip, where apparent shear rate as a function of plate edge shear stress $\left(\tau_{R}\right)$ is expressed as

$$
\dot{\gamma}_{\text {apparent }}=\dot{\gamma}_{\text {true }}\left(\tau_{R}, R\right)+\frac{2 v_{\text {slip }}\left(\tau_{R}\right)}{H} .
$$

The plate edge shear stress is given by

$$
\tau_{R}=\frac{M}{2 \pi R^{3}}\left[3+\frac{d \ln M}{d l \ln \dot{\gamma}_{\text {apparent }}(R)}\right],
$$

where $M$ is the measured torque and $R$ is the radius of the plate. The resulting slip lengths $\left(l_{\text {slip }}=\nu_{\text {slip }} / \dot{\gamma}_{\text {true }}\right)$ calculated from the shear rates $\left(\dot{\gamma}_{\text {true }}\right)$, and slip velocities $\left(\nu_{\text {slip }}\right)$ measured at plate edge stresses of $\tau_{R}=1,10,100$, and $1000 \mathrm{~Pa}$ for PCC(7)/PEG suspensions ( $\phi$ 

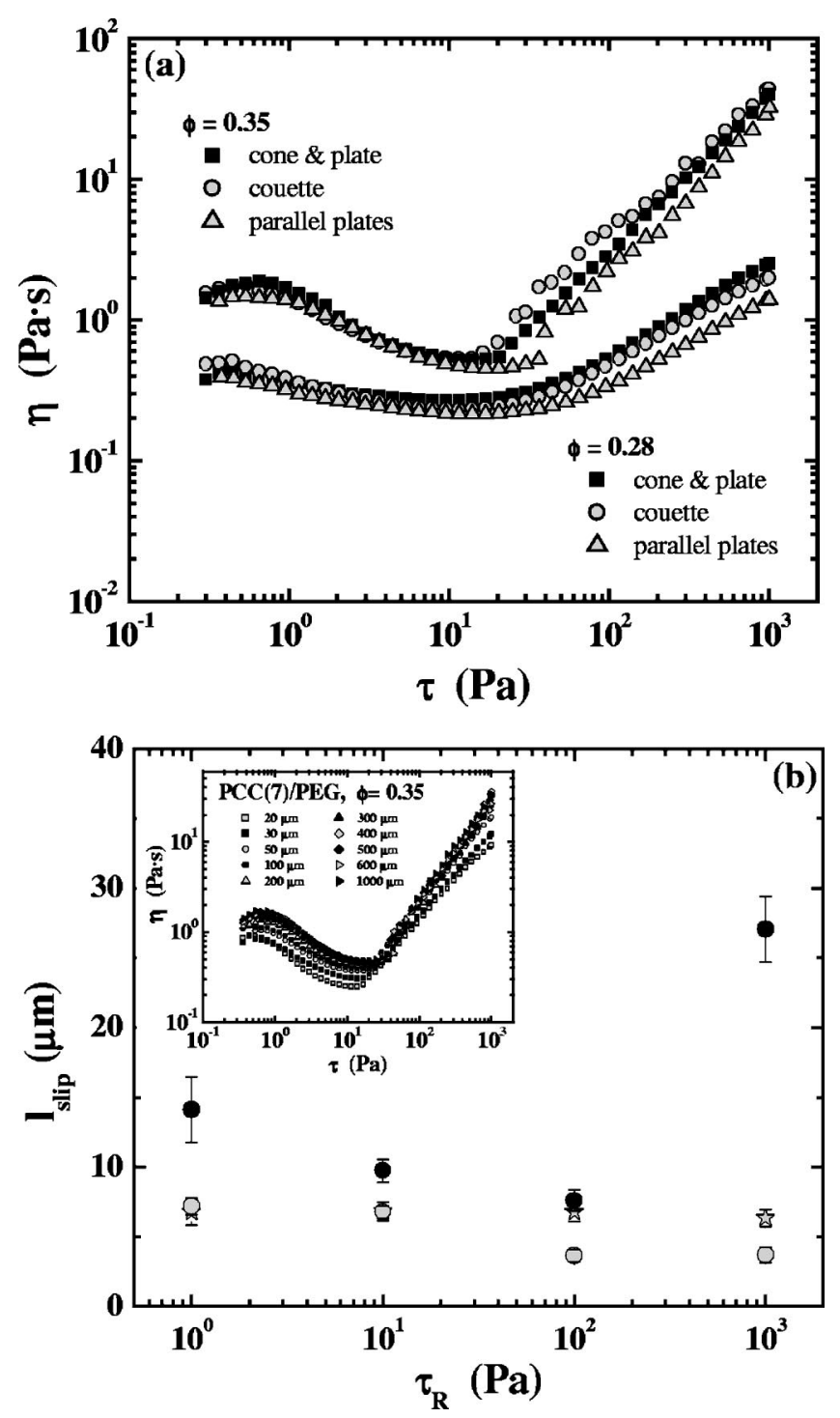

FIG. 4. Effects of tool geometry on the shear viscosity of $\mathrm{PCC}(7) / \mathrm{PEG}$ suspensions $(\phi=0.28$ and $\phi=0.35)$ : (a) comparison of cone $(25 \mathrm{~mm}, 0.02 \mathrm{rad})$, Couette [17 mm inner diameter (i.d.), $0.25 \mathrm{~mm}$ gap] and parallel plate (25 mm, $0.5 \mathrm{~mm}$ gap) geometries. (b) Plot of slip length determined for PCC(7)/PEG suspensions at $\phi=0.28$ (open circles), $\phi=0.35$ (filled circles), and a $5 \mathrm{~Pa}$ s oil standard (stars). The inset plot presents the parallel plate measurements of the PCC(7)/PEG, $\phi=0.35$ suspension at several gap heights.

$=0.28$ and $\phi=0.35)$ as well as for a $5 \mathrm{~Pa}$ s Newtonian oil reference are presented in Fig. 4(b). The measurements using the Newtonian oil calibration standard exhibited a slight dependence on gap as expected [Maranzano (2001); Henson and Mackay (1994)]. Slip lengths for the continuous shear thickening sample $(\phi=0.28)$ are comparable, indicating no significant slip. Slip is clearly significant for the discontinuous PCC(7)/PEG suspension, $(\phi=0.35)$, especially at high shear rates in the shear thickening regime. This observed slip is qualitatively similar to the observations of Maranzano (2001), where significant slip was observed for discontinuous shear thickening suspensions of spherical, 
colloidal particles in the shear thickening regime. These large increases in slip length in the shear thickening regime are thought to be associated with the formation of samplespanning hydroclusters responsible for jamming of the particles in the flow field [Maranzano (2001); Maranzano and Wagner(2001b)].

As observed from the variable gap, parallel plate rheology [Fig. 4(b) inset], reducing the gap dimension results in a slight reduction in the critical stress for shear thickening for this concentrated PCC/PEG suspension. This trend is similar to observations of concentrated, spherical colloidal suspensions exhibiting shear thickening previously reported by Chow and Zukoski (1995), but are not consistent with observations of [Maranzano (2001); Maranzano and Wagner (2001a)], also on spherical silica colloidal suspensions, which showed no gap size dependence on the critical stress for shear thickening.

Transient experiments were conducted using the controlled strain rheometer on the PCC(7)/PEG suspensions exhibiting continuous shear thickening behavior at a concentration just below the critical volume fraction for discontinuous shear thickening $(\phi$ $=0.30$ ). The steady shear rheology for the PCC(7)/PEG, $\phi=0.30$ suspension is presented in the upper inset graph in Fig. 5(a). Figure 5(a) presents the results of step rate experiments performed at an initial shear rate of $\dot{\gamma}=10 \mathrm{~s}^{-1}$ for $30 \mathrm{~s}$, followed by a step rate increase to $\dot{\gamma}=300 \mathrm{~s}^{-1}$ within the shear thickening regime, and back to the initial shear rate of $\dot{\gamma}=10 \mathrm{~s}^{-1}$ for $30 \mathrm{~s}$. The lower inset graph in Fig. 5(a) shows a magnified view of the step rate experiment local to the rate change transitions. Based on the results of this step rate experiment performed on the high particle aspect ratio PCC(7)/PEG suspension, it is clear that the response time for both step rate increases from the shear thinning regime into the shear thickening regime and back to the shear thinning regime is rapid. This is most evident for the latter case, where the step decrease in rate from $\dot{\gamma}$ $=300 \mathrm{~s}^{-1}$ to $\dot{\gamma}=10 \mathrm{~s}^{-1}$ demonstrates nearly instantaneous relaxation with little observable oscillatory behavior. These fast response times for transitioning from shear thinning to shear thickening and vice versa are also observed in suspensions of charge stabilized and near hard-sphere suspensions [Bender and Wagner (1996); Maranzano (2001); Maranzano and Wagner (2001a)].

The relaxation from shear thickening to shear thinning behavior is faster than the resolution capabilities of the fast data acquisition instrument $(5 \mathrm{~ms})$, suggesting that little microstructural rearrangements take place between the shear thickening and shear thinning state. This experimental result is contrary to expectations of the hypothesis that shear thickening in highly anisotropic particle suspensions results from the formation of "entanglements" or jammed networks of particles that rotate out of flow alignment. Such misalignment upon shear thickening and subsequent realignment upon returning to lower shear rates would require a finite relaxation time. Rather, the instantaneous stress response (on the experimental time scale) is consistent with a purely hydrodynamic contribution to the measured shear viscosity in the shear thickened state [O'Brien and Mackay (2000)].

Figure 5(b) presents the results of flow reversal experiments, plotted as reduced stress versus strain units $(t \dot{\gamma})$, performed at several shear rates for the PCC(7)/PEG, $\phi=0.30$ suspension. For flow reversal experiments conducted at rates below the critical rate for shear thickening (below $\dot{\gamma}=100 \mathrm{~s}^{-1}$ ) we observe a reasonable overlay of the resulting stress data when plotted against strain, which suggests particle rotation upon flow reversal. Within the shear thickening regime, we observe that the stress overshoots become increasingly pronounced up to a rate of $\dot{\gamma}=300 \mathrm{~s}^{-1}$. The flow reversals performed at higher shear rates exhibit less stress overshoot, with an apparent increase in the period of oscillation. Identical sets of flow reversal experiments performed on Newtonian oil standards exhibit no evidence of this positive stress overshoot. The observed stress under- 

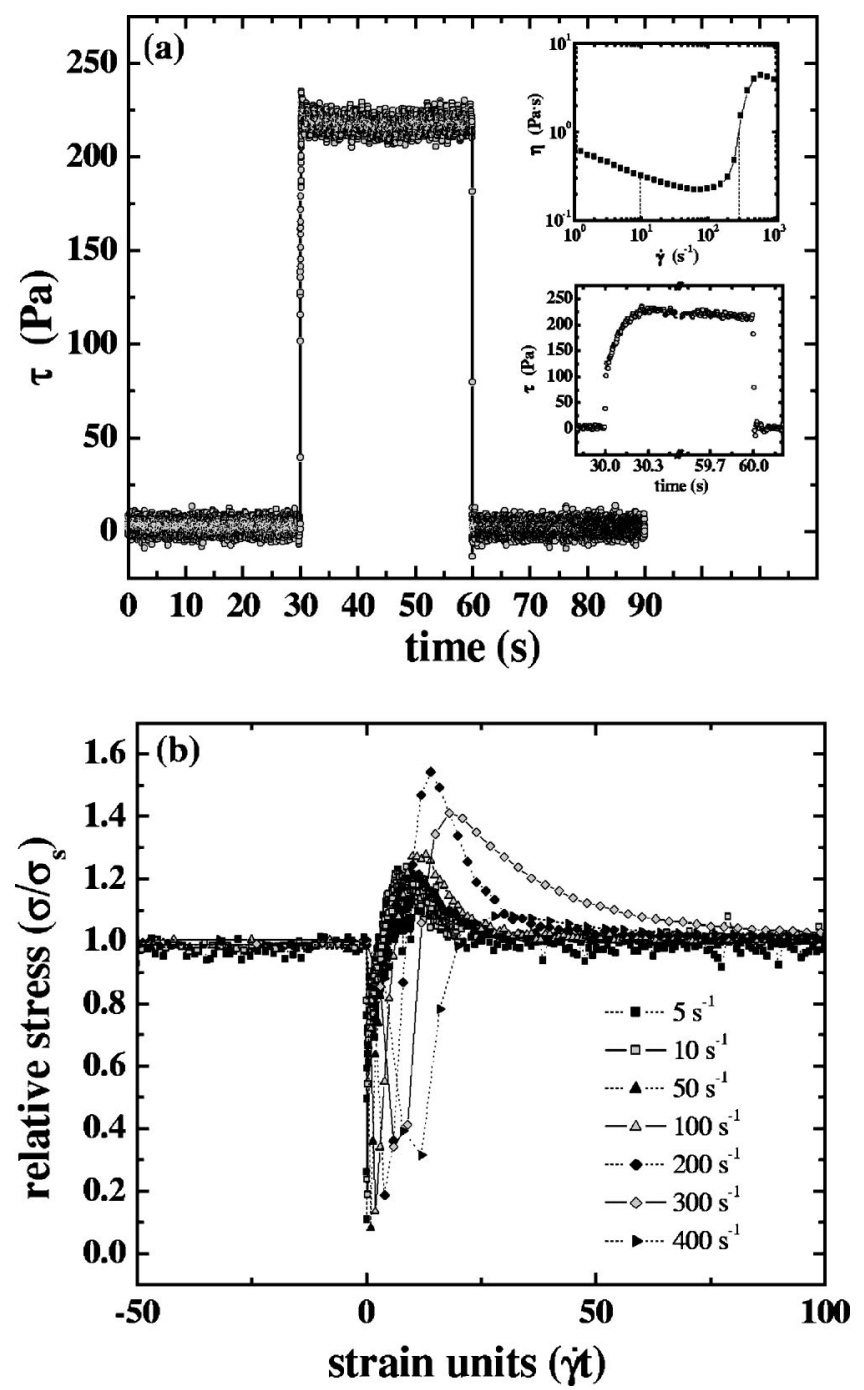

FIG. 5. (a) Step rate experiment performed on PCC(7)/PEG suspension at $\phi=0.30: \dot{\gamma}=10 \mathrm{~s}^{-1}$ for $30 \mathrm{~s}$ (shear thinning regime), $\dot{\gamma}=10 \mathrm{~s}^{-1}$ for $30 \mathrm{~s}$ (shear thickening regime), $\dot{\gamma}=10 \mathrm{~s}^{-1}$ for $30 \mathrm{~s}$ (shear thinning regime). Top inset plot shows steady shear rheology of PCC(7)/PEG at $\phi=0.30$. Bottom inset presents magnified data local to the point of step rate increase and step rate decrease. (b) The results of flow reversal experiments performed at several shear rates on the PCC(7)/PEG suspension at $\phi=0.30$.

shoots that precede stress overshoots observed in the PCC(7)/PEG, $\phi=0.30$ suspension were also observed during the experiments performed on the oils and can be assigned to instrument inertia effects.

Figure 6 presents results from ascending steady stress sweeps using the cone and plate $(0.02 \mathrm{rad}, 25 \mathrm{~mm})$ geometry for each of the PCC/PEG suspensions measured within 1 week following sample preparation. As illustrated in the flow curves in Fig. 6, each of the PCC/PEG suspensions clearly exhibit shear thickening behavior at high shear rates or applied shear stresses. Each of the suspensions exhibited little hysteresis between consecutive ascending and descending stress sweeps as shown earlier in Fig. 3(a) for the PCC(7)/PEG suspensions, confirming the reversible nature of the observed shear thick- 

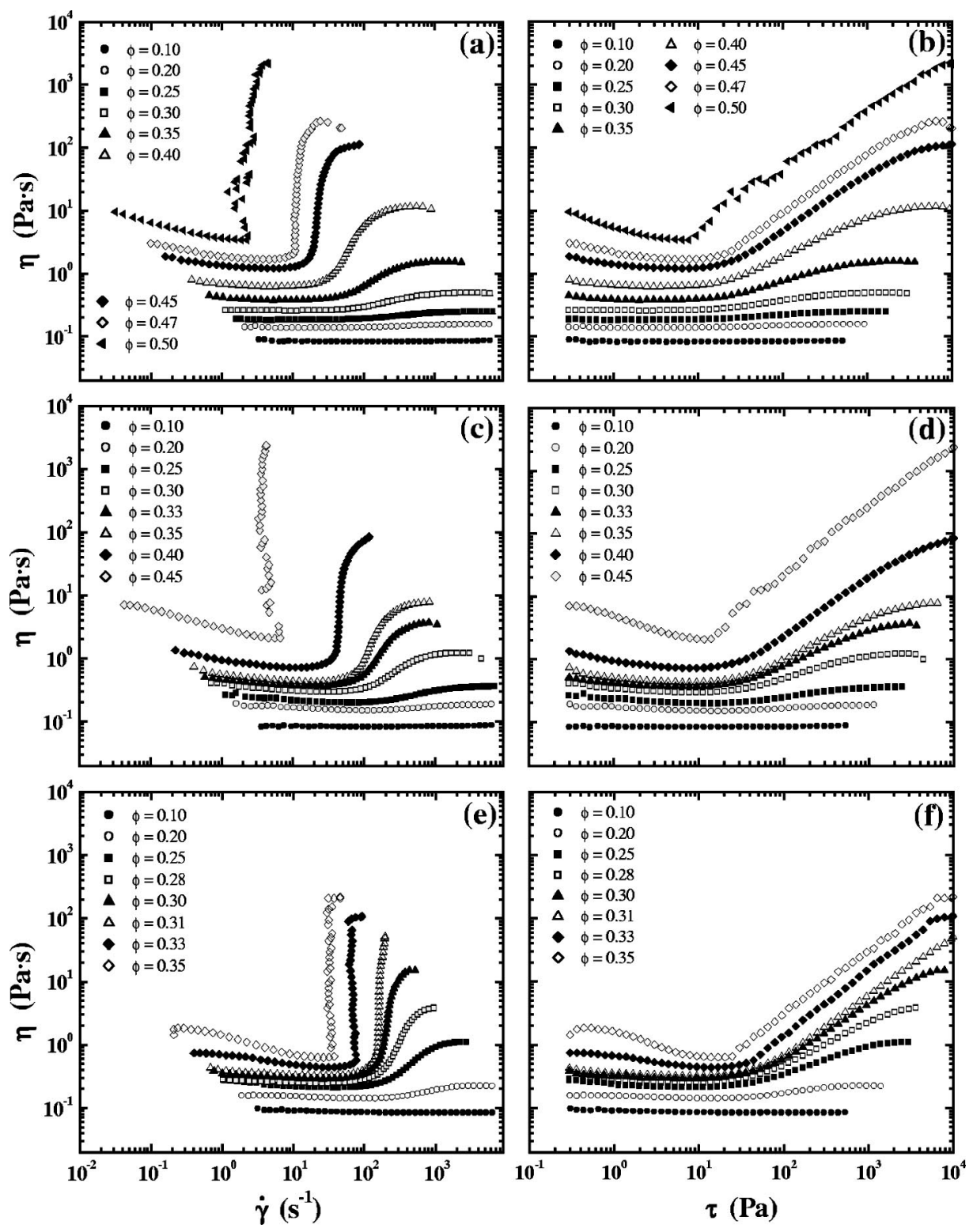

FIG. 6. Steady shear viscosity of PCC/PEG suspensions at several particle volume fractions. PCC(2)/PEG: (a) viscosity vs shear rate, (b) viscosity vs stress. PCC(4)/PEG: (c) viscosity vs shear rate, (d) viscosity vs stress. PCC(7)/PEG: (e) viscosity vs shear rate, (f) viscosity vs stress.

ening behavior for each type of suspension. Note that the values of the critical stress for shear thickening, defined as the minimum in the viscosity flow curve, are nearly independent of particle volume fraction and particle aspect ratio. As in spherical particle suspensions, each of these PCC suspensions demonstrates a distinct transition from continuous to discontinuous shear thickening behavior with increasing particle volume fraction. However, the extremely shear thickening samples still exhibit a relatively low vis- 
TABLE II. Maximum packing fraction $(\tau=1 \mathrm{~Pa})$, sedimentation volume fraction, and critical volume fraction for shear thickening transition for the PCC/PEG suspensions.

\begin{tabular}{lccc}
\hline \hline & PCC (2:1)/PEG & PCC(4:1)/PEG & PCC(7:1) PEG \\
\hline$\phi_{\text {sed }}$ & 0.614 & 0.598 & 0.596 \\
$\phi_{\max }(1 \mathrm{~Pa})$ & 0.562 & 0.519 & 0.515 \\
$\phi_{c}$ & 0.454 & 0.378 & 0.308 \\
\hline \hline
\end{tabular}

cosity at low shear rates, which is in contrast to observations for spherical particle suspensions that often exhibit a yielding behavior at the high volume fractions required for observing discontinuous shear thickening.

The "critical volume fraction," $\phi_{c}$, for the onset of discontinuous shear thickening was determined for each of the suspensions as the particle volume fraction when $\eta \propto \tau^{1}$ in the shear thickening region; the value for $\phi_{c}$ for each type of suspension is listed in Table II. Figure 7 presents $\phi_{c}$ for the anisotropic particle PCC/PEG suspensions as well as for the surface charge neutralized (near hard sphere) particle suspensions (aspect ratio=1) reported by Maranzano (2001). As observed from the steady shear rheology measured for these systems, $\phi_{c}$ decreases with increasing particle aspect ratio.

The correspondence between the onset of discontinuous shear thickening and predicted phase transitions has been noted for near hard sphere suspensions by O'Brien and Mackay (2000), but no physical connection has been established. Hence, for comparison, Fig. 7 includes references such as the hard-sphere phase transition from fluid to crystal $(\phi=0.5)$ and the isotropic-nematic phase transition for fluids of monodisperse prolate

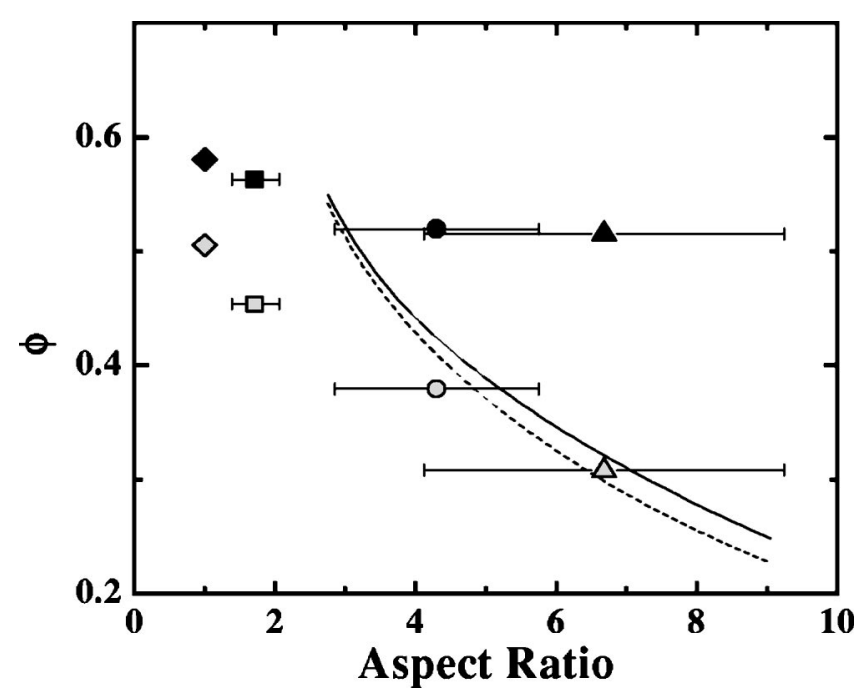

FIG. 7. Critical volume fraction for discontinuous shear thickening: PCC(2)/PEG (open square), PCC(4)/PEG (open circle), PCC(7)/PEG (open triangle), and hard sphere suspension [Maranzano (2001)] (open diamond). Low shear $(\tau=1 \mathrm{~Pa})$ maximum packing fraction for PCC(2)/PEG (filled square), PCC(4)/PEG (filled circle), and PCC(7)/PEG (filled triangle) suspensions determined from fits in Fig. 8. Also plotted is the maximum packing fraction for hard spheres [Phan et al. (1996); Pusey and van Megen (1987)], (filled diamond). Lines on the graph represent isotropic (dash line) and nematic (solid line) phase boundaries based on computer simulations of ellipsoidal particle fluids [Lee (1988); Camp et al. (1996)]. 


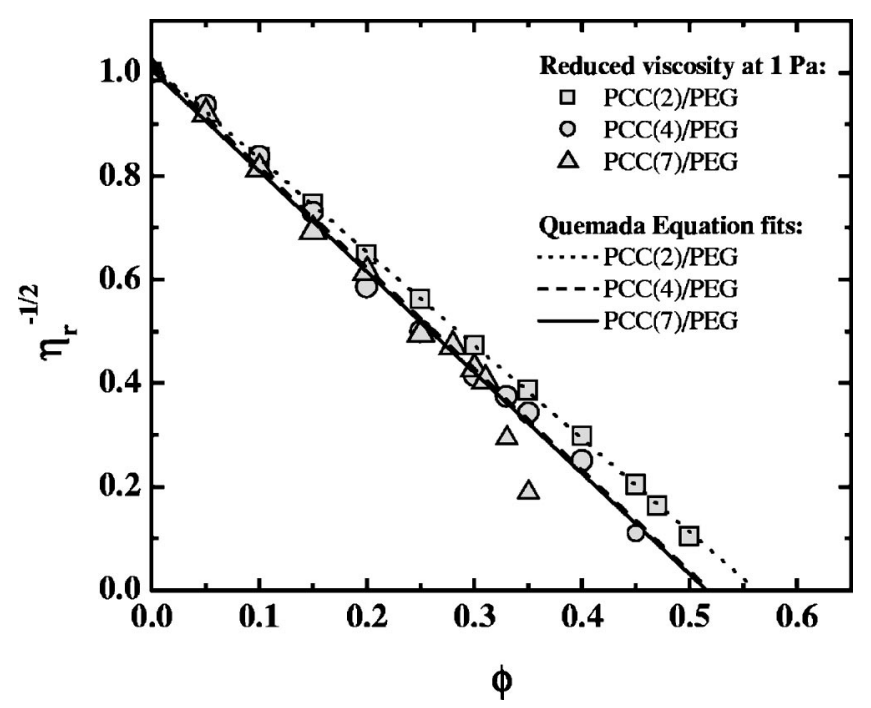

FIG. 8. Low shear data $(\tau=1 \mathrm{~Pa})$ from steady stress sweep experiments on PCC/PEG suspensions plotted according to Eq. (3) vs particle volume fraction. Lines represent fits to the data at volume fractions below $\phi_{c}$ and are extrapolated to determine $\phi_{\max }$.

ellipsoids, as calculated from computer simulations by Camp et al. (1996) and Lee (1988). Note that the simulations of Lee (1988) have determined that this phase transition disappears below an aspect ratio of 2.75 .

For spherical particles, the volume fraction at the glass transition was experimentally determined by Pusey and van Megen (1987) $(\phi=0.58)$. This closely corresponds to the maximum packing fraction $\phi_{\max }$ determined experimentally by analyzing the relative zero-shear viscosity using the Krieger-Dougherty-Quemada equation [Eq. (3)] for near hard sphere suspensions by Phan et al. (1996):

$$
\eta_{r}=\left(1-\frac{\phi}{\phi_{\max }}\right)^{-2}
$$

A similar analysis of the low shear viscosity was performed for the PCC/PEG suspensions, as shown in Fig. 8. Due to the relatively large size of the particles, true, zero-shear viscosities were not obtained. Figure 8 presents stress sweep data at $\tau=1 \mathrm{~Pa}$ for each system plotted as $\left(\eta_{r}^{-1 / 2}\right)$ vs $\phi$, where $\eta_{r}$ is the relative viscosity. The lines in Fig. 8 represent linear fits to the data of each PCC/PEG suspension at volume fractions below the critical volume fraction for discontinuous shear thickening, $\phi_{c}$. The intercepts of the linear fits with the abscissa give the maximum packing fractions $\phi_{\max }$, which are presented in Table II and included in Fig. 7. It is notable that despite the differences in anisotropy, the maximum packing fractions determined at $\tau=1 \mathrm{~Pa}$ by fitting data within the shear thinning regime are comparable for each aspect ratio. At volume fractions above $\phi_{c}$, we observe that the higher particle aspect ratio suspensions (being particularly evident for the PCC(7)/PEG suspension above $\phi=0.31$ ) exhibit deviation from the linear fits (which were generated from data below $\phi_{c}$ ). For reference, the sedimentation volume fractions are also presented in Table II, which were determined for each of the PCC suspensions through measurement of solids concentration in the sediment obtained from centrifugation $(2 \mathrm{~h}$ at $1750 \mathrm{~g})$. These volume fractions are higher than the maximum packing fractions for shear flow, and are also only weakly dependent on aspect ratio. 


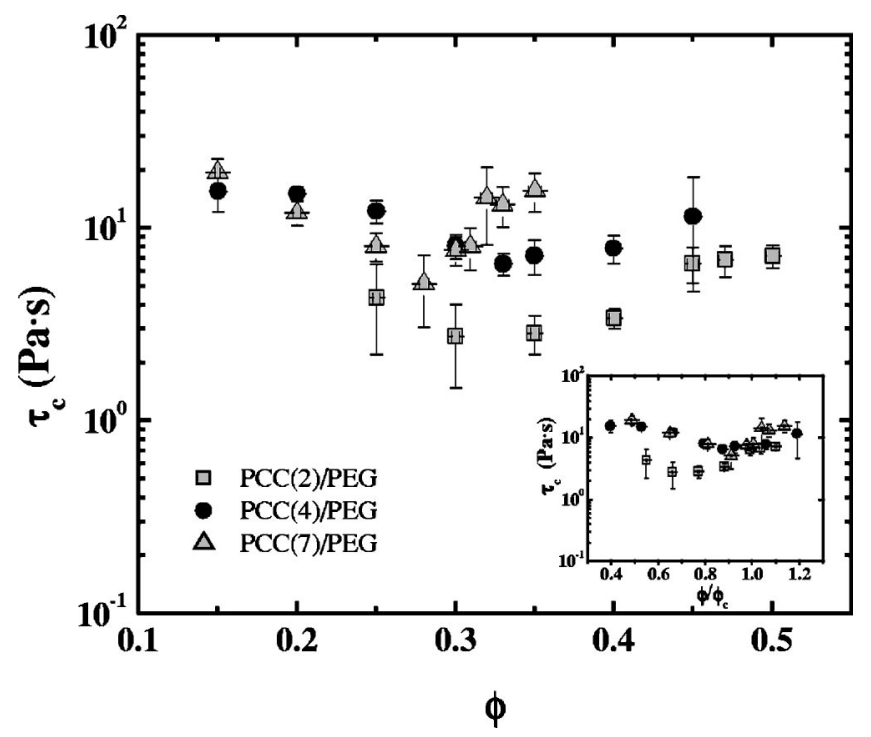

FIG. 9. Critical stress for shear thickening, $\tau_{c}$ for $\mathrm{PCC}(2) / \mathrm{PEG}, \mathrm{PCC}(4) / \mathrm{PEG}$, and $\mathrm{PCC}(7) / \mathrm{PEG}$ suspensions at several volume fractions. Inset plot presents critical stress, $\tau_{c}$ vs $\phi / \phi_{c}$.

These observations are consistent if the particles are highly aligned (such as in a nematic phase) at high packing fractions. Note that measurements of the random unoriented packing fraction for rod-like particles [Philipse (1996)] are much lower than these volume fractions.

The critical stress values for the onset of shear thickening $\tau_{c}$ were obtained from the minima of quadratic fits to $\log (\eta)$ vs $\log (\tau)$ data local to the critical stress region [Maranzano (2001); Maranzano and Wagner (2001a, 2001b)]. Figure 9 presents the critical stress for shear thickening of each of the PCC/PEG suspensions at several volume fractions. Critical stress values determined for each of the suspensions demonstrate only minor variation with particle concentration. The critical stresses decrease with increasing particle loading until the critical volume fraction is reached; thereafter, they begin to increase slightly in magnitude. Despite distinct differences in particle anisotropy, the critical stress values determined for each of the PCC/PEG suspension are close in magnitude, demonstrating a slight increase in value with increasing particle aspect ratio. In fact, as will be shown, the trend can be understood due to differences in the minor axis dimension of the particle.

\section{B. SANS investigation of precipitated calcium carbonate suspensions}

Figure 10 presents the static scattering results in the form of absolute scattering intensity $(I)$ versus scattering vector amplitude $(q)$ for PCC(7)/PEG suspensions at several volume fractions. For the range of momentum transfer accessible in the SANS experiments, the scattering is within the Porod regime $(q \mathrm{D}>1)$, such that the observed scattering intensity is from the particle-solvent interface. The intensity exhibits the expected power law of $q^{-4}$ for Porod regime scattering from objects with sharp interfaces. Consequently, these SANS experiments cannot directly determine the particle size and shape distribution; however, they can be used to directly measure particle alignment [Kernick and Wagner (1999)]. Scattering patterns resulting from samples having particle loadings below $\phi=0.29$ were consistently isotropic, indicating that the particles are randomly 


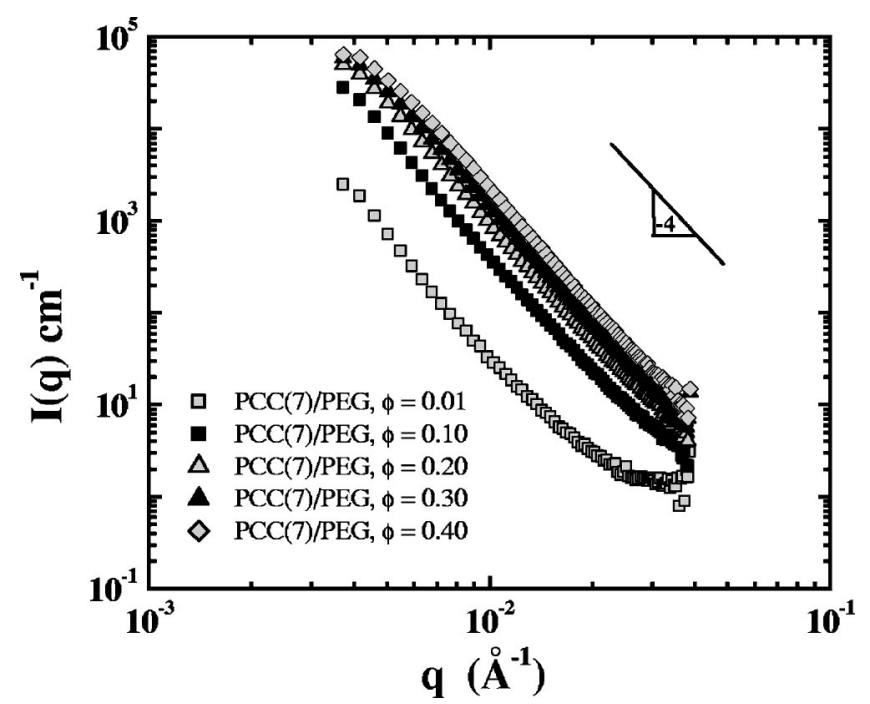

FIG. 10. Circularly averaged static SANS data plotted as absolute intensity $(I)$ vs scattering vector magnitude (q) for PCC(7)/PEG suspensions at several particle concentrations.

oriented within these suspensions. Several of the samples having particle loadings above $\phi=0.29$ (at volume fractions near and above the critical volume fraction for discontinuous shear thickening, $\phi \sim 0.31$ ) exhibit weakly anisotropic scattering. This suggests that particles and possibly nematic particle domains became locally aligned as a result of sample loading, and the induced alignment was able to persist following sample preparation and during scattering experiments $(5 \mathrm{~min})$.

PCC(7)/PEG suspensions with volume fractions of $\phi=0.29$ and $\phi=0.25$, which exhibit continuous shear thickening, were investigated with flow-SANS. Scattering data was collected at shear rates of $1,10,30,100,200$, and $800 \mathrm{~s}^{-1}$ for both concentrations. These shear rates correspond to characteristic rheological signatures; low shear rates within the shear thinning regime, near the shear thickening transition $\left(\dot{\gamma}_{c} \sim 30 \mathrm{~s}^{-1}\right.$ for PCC(7)/PEG, $\phi=0.29)$, within the shear thickening regime and near the observed high shear plateau region $\left(\dot{\gamma}_{c}>\sim 500 \mathrm{~s}^{-1}\right.$ for PCC(7)/PEG, $\left.\phi=0.29\right)$. Figure 11(a) presents the rheology of these samples measured on the SR 5000 stress control rheometer using the 25 $\mathrm{mm}, 0.02 \mathrm{rad}$ cone prior to testing in the flow-SANS Couette cell. Scattering patterns resulting from radially and tangentially directed neutron beam experiments (see Fig. 2) for the $\phi=0.29, \mathrm{PCC}(7) / \mathrm{PEG}$ suspension at the shear rates investigated are presented in Figs. 11(c) and 11(d).

The anisotropy of the radial scattering measurements (velocity-vorticity plane) suggest a high degree of particle orientation with flow direction at all shear rates investigated. The isotropic tangential patterns (velocity gradient-vorticity plane) are also consistent with long-axis flow alignment. The anisotropy in radial scattering measurements gradually increases and then decreases with increasing shear rate. In Fig. 11(b), radial scattering data at $q=0.01 \AA^{-1}$ is presented as a function of angle in the plane of the detector for the continuously shear thickening PCC(7)/PEG, $\phi=0.29$ sample at each shear rate interrogated.

A Rheo-SANS experiment was also conducted on a PCC(7)/PEG dispersion at a higher particle concentration of $\phi=0.35$. Due to the discontinuous shear thickening nature of this suspension, a controlled stress rheometer (Paar Physica, model UDS 200) was 

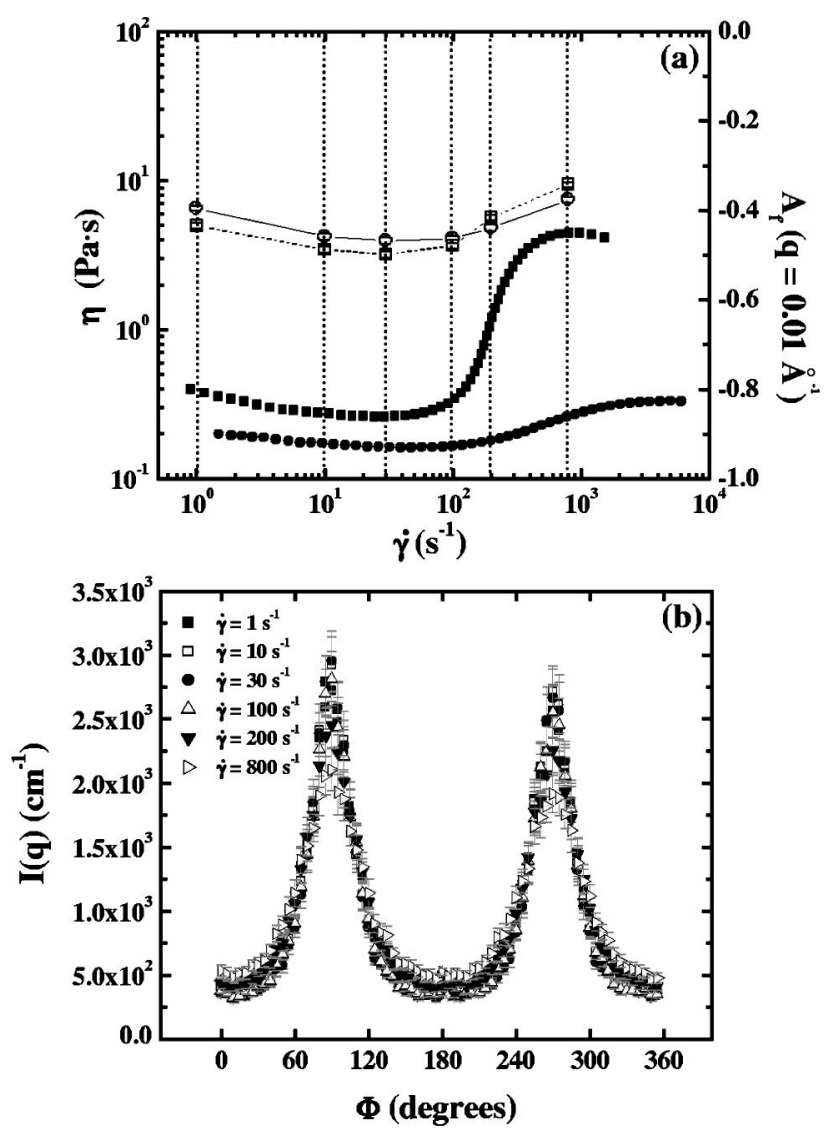

(c) Radial (x-velocity, y-vorticity)

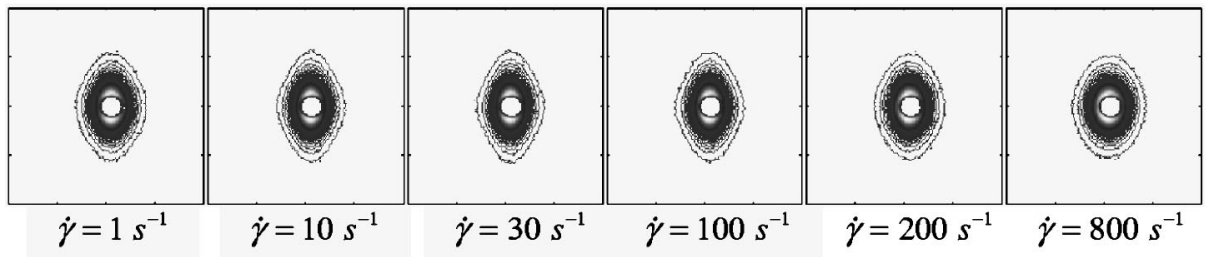

(d) Tangential (x-velocity gradient, $y$-vorticity)

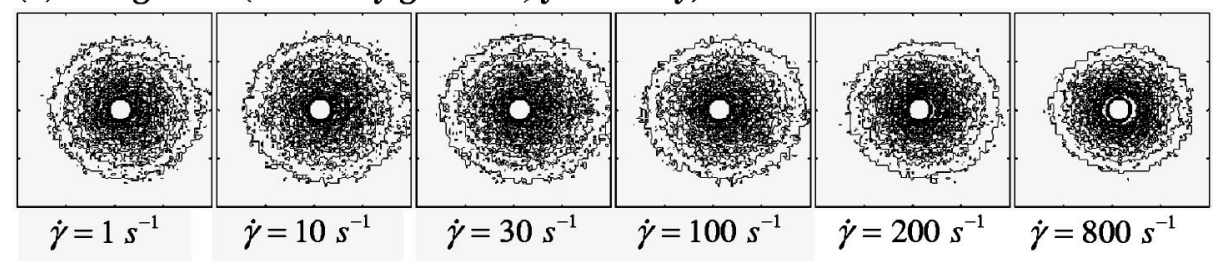

FIG. 11. (a) Alignment factors for PCC(7)/PEG suspensions, $\phi=0.25$ (open circle), $\phi=0.29$ (open square), and corresponding shear rheology, $\phi=0.25$ (filled circle), $\phi=0.29$ (filled square) at several shear rates. (b) Annular averaged scattering intensity $\left(q=0.010 \AA^{-1}\right.$, pixel width $\left.=4\right)$ for PCC $(7: 1) / \mathrm{PEG}$ dispersion at $\phi=0.29$. (c) Radial and (d) tangential scattering patterns resulting from flow-SANS investigation of a PCC(7)/PEG suspension at $\phi=0.29\left(q_{\min }=0.003 \AA^{-1}, q_{\max }=0.03 \AA^{-1}\right)$. 
required. The rheology of the suspension, presented in Fig. 12(a), was recorded concurrent with the collection of scattering data at applied stress values of 1, 4, 15, 30, 100, and $500 \mathrm{~Pa}$. The discontinuous shear thickening transition is observed to occur near $11 \mathrm{~Pa}$, which is consistent with measurements in our laboratory (Paar Physica, model MDS500, Couette geometry with $26.66 \mathrm{~mm}$ bob diameter), which are presented Fig. 12(a) for comparison. Figures 12(c) and 12(d) present the radial and tangential scattering patterns recorded during the experiment. This sample demonstrated yielding behavior in the Rheo-SANS shear cell at stresses below $1 \mathrm{~Pa}$. Figure 12(b) presents the annular averaged intensity versus angle from the scattering results recorded at each of the applied stresses. These results clearly demonstrate that a significant degree of particle alignment with flow direction is maintained during shear thinning as well as discontinuous shear thickening.

An illustration of the average particle alignment deduced from both two-dimensional scattering projections is provided in Fig. 2, which is consistent with the known behavior of flow-aligning nematics. Scattering from the radially directed incident beam corresponds to particles having long axis orientation about a director parallel to the flow direction. Scattering in the tangential direction is isotropic, as particle long axis alignment is distributed about a director parallel to the neutron beam direction.

The extent of long-axis alignment with the flow can be quantified through calculation of an alignment factor [Walker and Wagner (1996)] defined as

$$
A_{f}(q)=\frac{\int_{0}^{2 \pi} I(q, \Phi) \cos (2 \Phi) d \Phi}{\int_{0}^{2 \pi} I(q, \Phi) d \Phi} .
$$

In the earlier expression, $I(q, \Phi)$ is the scattering intensity observed in the plane of the detector in a cylindrical coordinate frame centered on the beam, where orientation angle $\Phi=0$ along the flow direction for the case of the radially oriented beam. The alignment factor $A_{f}$ can vary in value from -1 to 1 depending on the alignment of the particles relative to the shear field. For this arrangement of the Rheo-SANS experiments (beam and cell orientation, and scattering in the Porod limit), positive values for $A_{f}$ would be obtained for a system of prolate-like particles having orientation predominantly perpendicular to the flow direction, negative $A_{f}$ values would result from alignment predominately parallel to the flow direction, and alignment factor values near zero would represent an isotropic arrangement (random orientation) of anisotropic particles within the suspension. Figures 11(a) and 12(a) show the calculated alignment factors obtained from the data in Figs. 11(b) and 12(b) for direct comparison to the measured shear viscosity.

For the continuous shear thickening samples $[\phi=0.25, \phi=0.29$, Fig. 11(a)], we observe that the degree of particle alignment (as measured by $\left|A_{f}\right|$ ) increases with increasing shear rate within the shear thinning regime. Particles attain the greatest degree of flow alignment $\left|A_{f}\right|$ just prior to the shear thickening transition. Within the shear thickening regime, the flow alignment decreases slightly with increasing shear rate. The correlation between viscosity and shear alignment in the shear thinning regime is qualitatively understood as flow alignment reducing interparticle interactions in the flowing suspension. However, substantial alignment persists in the shear thickening regime up to the highest shear rate investigated $\left(800 \mathrm{~s}^{-1}\right)$. For the $\phi=0.29$ sample, the shear rate of $800 \mathrm{~s}^{-1}$ corresponds to the apparent high shear plateau in the viscosity. Most significant for understanding the microstructural basis for the observed shear thickening is the lack of quantitative connection between alignment factor and viscosity across the entire flow curve. 

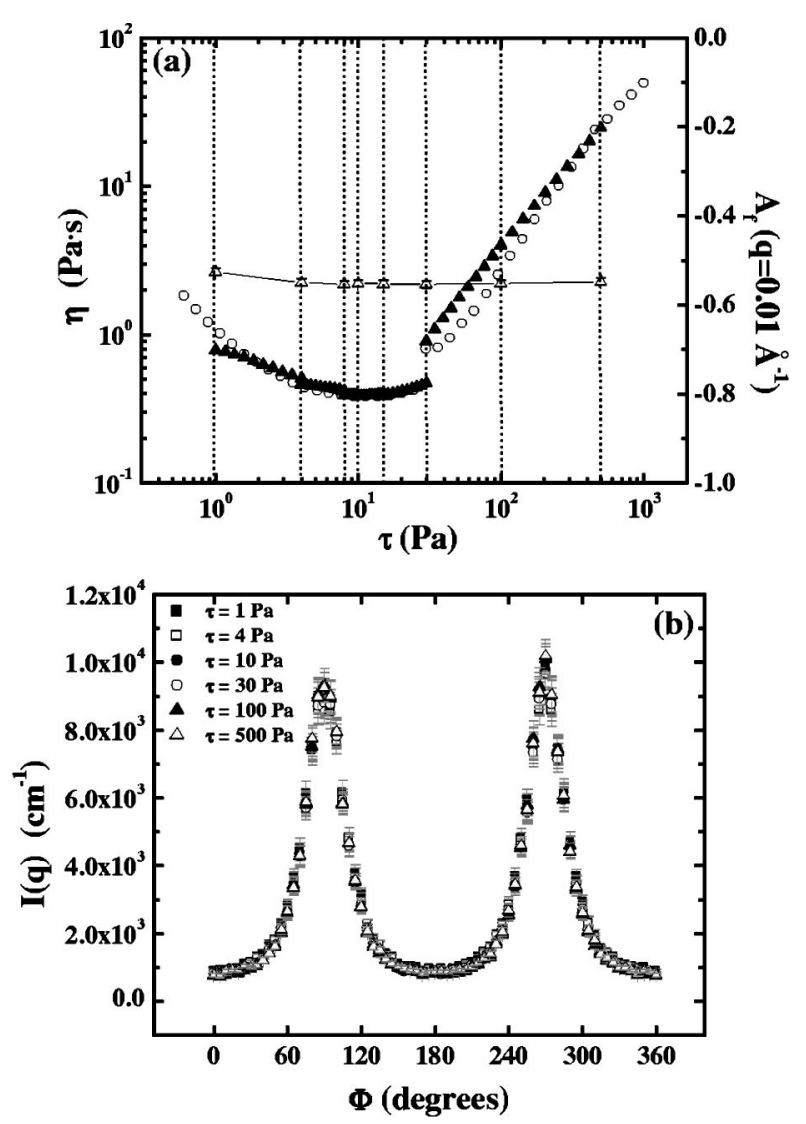

(c) Radial (x-velocity, y-vorticity)

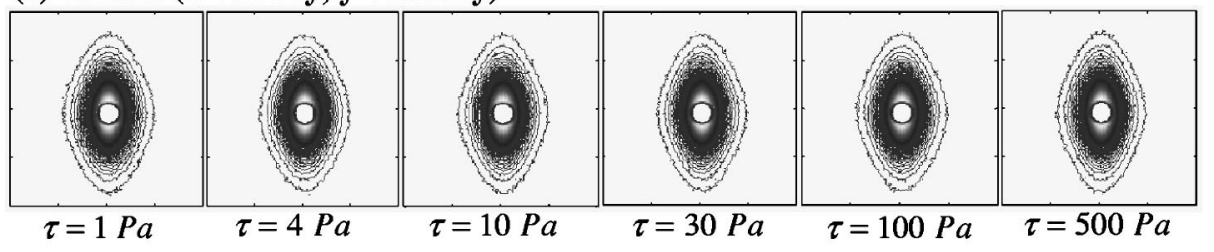

(d) Tangential (x-velocity gradient, $y$-vorticity)
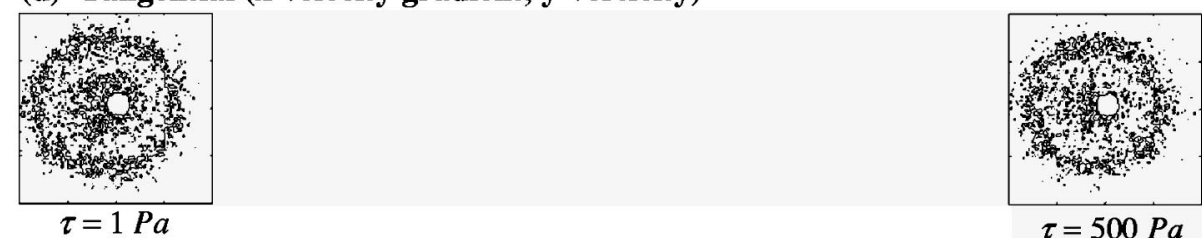

$\tau=500 P a$

FIG. 12. (a) Alignment factors for PCC(7)/PEG, $\phi=0.35$ dispersion (open triangles), the corresponding shear rheology measured in the Rheo-SANS shear cell (filled triangles), and the rheology measured using the laboratory couette (28.92 mm cup i.d.) geometry (open circles) at several stresses. (b) Annular averaged scattering intensity $\left(q=0.010 \AA^{-1}\right.$, pixel width=4) for PCC(7:1)/PEG dispersion at $\phi=0.35$. (c) Radial and (d) tangential scattering patterns resulting from Rheo-SANS investigation of the PCC(7)/PEG suspension at $\phi=0.35\left(q_{\min }\right.$ $=0.003 \AA^{-1}, q_{\max }=0.03 \AA^{-1}$ ). 
$\left|A_{f}\right|$ values at the lowest and highest shear rates measured are comparable, yet the viscosity at high shear rates is $\sim 10$ times higher and the stresses $\sim 10,000$ time greater for the latter state point. This suggests that the shear thickening response at high shear rates does not arise from the same forces that connect the microstructure to the stresses at low shear rates.

In Fig. 12(a), the calculated alignment factors for the discontinuous shear thickening sample $(\phi=0.35)$ again illustrates that the degree of particle long axis alignment with flow direction is greatest near the shear thickening transition. Unlike the continuous shear thickening samples, however, increases in applied stress beyond the critical stress (during discontinuous shear thickening) have little effect on the high degree of particle orientation. This demonstrates that discontinuous shear thickening in these suspensions of prolate particles during simple shearing flows is not the result of an entangling event associated with particles rotating out of flow alignment and forming a network of randomly oriented particles. Rather, we provide evidence that particle flow alignment remains constant through the discontinuous shear thickening transition.

\section{DISCUSSION}

Qualitatively, the acicular PCC/PEG suspensions exhibit many rheological traits that are similar to that of colloidal suspensions of spherical particles, including increasing degrees of shear thinning and shear thickening as particle volume fraction is increased. Reversible shear thickening is evident for all particle aspect ratios. At high applied stresses, we clearly observe the transition from continuous shear thickening behavior to discontinuous shear thickening as particle volume fraction is increased. Finally, transient rheological step-up and step-down measurements demonstrate that the transition in and out of the shear thickening regime is instantaneous on the time scale of the measurement.

Varying aspect ratio leads to distinct trends in the quantitative properties. Most significantly, the volume fraction at the transition from continuous to discontinuous shear thickening decreases with increasing particle aspect ratio. As a consequence, the anisotropic particle suspensions have lower, low shear viscosities than spherical particle suspensions at $\phi_{c}$. Flow reversals show transients that scale with strain, which is indicative of flow reorientation of the particles. Flow orientation of the particles is consistent with the observed low shear rheology.

Based on these rheological observations alone, it is not possible that flow induced microstructural changes from flow-alignment to a misaligned, potentially "entangled" network occur during the shear thickening transition. Although the shear-thinning rheology and its volume fraction dependence is consistent with flow alignment of the particles prior to shear thickening, the lack of significant hysteresis in the ascending and descending stress sweeps, as well as the instantaneous transient rheological response for jumps in and out of the shear thickening regime are inconsistent with a mechanism that requires significant reorientation of the particles. The entanglement network formation suggested in the literature should result in measurable transients and hysteresis. Finally, there are no significant qualitative differences in rheology in comparing the 2:1, 4:1, and 7:1 aspect ratio particles to that of spherical particles, which suggests the mechanism of shear thickening is not dependent on particle shape.

Our flow- and Rheo-SANS investigations clearly refute the hypothesis that particle misalignment and entanglement are responsible for shear thickening in suspensions of ellipsoidal particles having aspect ratios near those of the PCC particles investigated. Particles are observed to align with the flow, with increasing flow alignment corresponding to weak shear thinning in the suspension viscosity. As is particularly evident for the 
discontinuous shear thickening PCC(7)/PEG dispersion $(\phi=0.35)$, the particles maintain a high degree of flow-oriented alignment during shear thickening and throughout the shear thickened regime. At lower volume fractions where continuous shear thickening is observed, there is a weak reduction in alignment at high stresses. However, quantitative comparison of the alignment factors, $\left|A_{f}\right|$ and the measured viscosity demonstrates that the shear thickening is not a direct consequence of reduced flow alignment of the particles as the viscosity is much higher at the higher shear rates for points on the flow curve with equal alignment factors.

One could postulate, however, that the reduced alignment in the continuous shear thickening regime consists of a heterogeneous dispersion microstructure composed of regions of highly aligned and highly misaligned (entangled) particles. These regions would be averaged in the SANS measurements to give only moderate changes in net alignment. However, this would be inconsistent with the measurements for the discontinuous shear thickening, which would be expected to be even more heterogeneous given the magnitude of the rheological response. Further, although such misalignment might be expected to lead to a type of jamming and increased viscosity for high aspect ratio suspensions, it is difficult to envision how this mechanism would operate for only slightly anisotropic particle suspensions, such as the PCC(2)/PEG suspensions studied here. The continuously evolving rheological behavior from spherical to slightly anisotropic $(L / D$ $\sim 2$ ) and eventually, highly anisotropic particle suspensions $(L / D \sim 7)$ is fundamentally inconsistent with the hypothesis of flow-misalignment driving shear thickening.

A consistent understanding of shear thickening in anisotropic particle suspensions that can account for all of the experimental observations is provided by the established mechanism of shear thickening for colloidal suspensions of spherical particles, namely, the formation of lubrication hydroclusters as a consequence of the dominance of lubrication hydrodynamic interactions between particles at high shear rates. As has been shown by rheological investigations, neutron scattering, flow dichroism, and simulations, reversible shear thickening in concentrated colloidal suspensions of spherical particles is a consequence of short-range lubrication forces correlating particle motion such that "hydroclusters" form, which lead to increased hydrodynamic dissipation in the shearing suspension. Comparison suggests that shear thickening in our prolate anisotropic suspensions is also the result of hydrocluster formation in the flow-aligned state. Discontinuous shear thickening results from "jamming" or percolation of the hydroclusters across the flow field. Our slip measurements show significant increase in slip length in the discontinuous shear thickening state, which is also a signature of hydrocluster formation [Maranzano (2001); Maranzano and Wagner (2001a, 2001b)]. The rapid transient response for these concentrated suspensions of anisometric particles in the shear thickening regime is a signature that the stresses are dominated by hydrodynamic interactions rather than direct interparticle forces [O'Brien and Mackay (2000); Bender and Wagner (1995); D'Haene et al. (1992)]. Stresses due to hydrodynamic interactions relax instantaneously upon flow cessation (on the experimental time scale) and do not require particle diffusion or reorientation. Further, because there is a clear lack of correspondence between the stresses and the degree of particle alignment in the shear thickening regime, the stresses at these high shear rates are not directly associated with interparticle forces that directly depend on the particle alignment, as would be the case for electrostatic, steric, or van der Waals interparticle interactions.

Shear thickening by hydrocluster formation in the PCC/PEG suspensions does not require significant microstructure rearrangement or changes in particle alignment. Although our SANS measurements are limited experimentally to the high $q$ limit (Porod regime), which renders them insensitive to the small change in interparticle spacing 
associated with hydrocluster formation, they are highly sensitive to particle alignment, which is observed to be maintained through discontinuous shear thickening. Importantly, the formation of hydroclusters that bridge across the flow field is expected to be easier for ellipsoidal shaped particles in comparison to spherical particles. Flow-aligned prolate ellipsoids are expected to organize into a "stack" spanning the flow field at lower particle volume fractions than spherical particles, as the aligned ellipsoids interact over a much more significant contact area (a 2- $d$ line) as opposed to a 1- $d$ point contact as for spherical particles. Consequently, the volume fraction required to percolate hydroclusters and generate discontinuous shear thickening is qualitatively expected to decrease with increasing particle aspect ratio, in agreement with experimental observation.

The reduction in the degree of flow alignment that is observed during continuous shear thickening in the PCC(7)/PEG suspensions is also to be expected if hydroclusters are the source of the increased energy dissipation during flow. Simulations have shown the dynamic nature of hydrocluster formation in spherical particle suspensions. At volume fractions below the threshold for hydrocluster percolation, i.e., in the regime of continuous shear thickening, hydroclusters are expected to be continuously forming and dissipating. Note that slip measurements in this regime do not show significant wall slip, suggesting such clusters are small relative to the tooling gap. Transient hydroclusters of elliptical particles will move coherently as an aligned stack or "bundle" for some limited time in the flow field. However, because such hydroclusters are expected to have a lower aspect ratio than that of the individual particles, they will be less-flow aligning than the primary particles of which they are composed. These clusters of particles will continue to be less flow aligning than the particles from which they form unless sufficient particle loading exists to cause the clusters "jam" or percolate the flow field such as for discontinuous shear thickening. Consequently, the slight reduction in the degree of flow alignment as observed in the continuous shear thickened state is consistent with hydrocluster formation.

The conclusion that shear thickening in these acicular suspensions is a consequence of hydrocluster formation can be tested by a quantitative comparison of the stresses required to initiate shear thickening with the reported results for spherical particle suspensions. As the particles are highly flow aligned through the shear thickening transition, the relevant particle dimension to characterize particle hydrodynamic interactions between flowaligned ellipsoids will be the minor axis. When viewed along streamlines, aligned ellipsoids have a cross section the same as a sphere of diameter equal to the minor axis (see Fig. 2). Consistent with this picture are the experimental observations that the particle aspect ratio will affect its propensity to flow align and the volume fraction at which discontinuous shear thickening appears, but not the critical stress required to initiate shear thickening at a given volume fraction.

Investigations of suspensions of surface charge neutralized spherical silica particles ranging over a decade in particle size by Maranzano (2001) and Maranzano and Wagner (2001b) validated the theoretical prediction that the critical stress for shear thickening scales inversely with the cube of particle radius for hard-sphere suspensions. In Fig. 13, we present the results of critical stress versus minor axis dimension (radius) for each of the PCC/PEG suspensions, as well as the data and linear regression obtained by Maranzano (2001) through fitting critical stress versus particle radius for the near hard-sphere suspensions. This figure clearly illustrates that the critical stress for shear thickening can be predicted for the anisotropic particle PCC/PEG suspensions based on the minor axis particle dimension. Despite large differences in the average length and particle aspect 


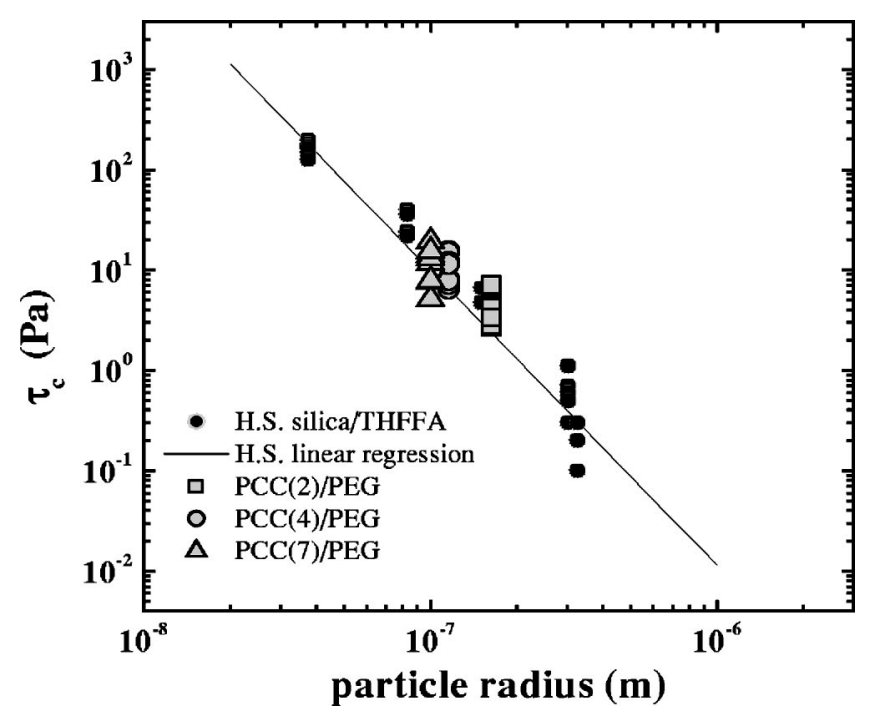

FIG. 13. Comparison of the critical stress values measured for PCC/PEG suspensions (open symbols) with those measured for near hard sphere suspensions (filled circles) [Maranzano (2001); Maranzano and Wagner (2001a)]. Line represents linear regression of surface charge neutralized, near hard sphere critical stress data. Particle radius corresponds to the minor axis for the acicular PCC particles.

ratio, the average PCC particle diameters are quite similar, as presented in Table I. This scaling of the critical stress for the PCC/PEG suspensions is consistent with the mechanism of shear thickening through hydrocluster formation.

Previous research on discontinuous shear thickening in spherical particle suspensions [O'Brien and Mackay (2000)] suggests that the particle volume fraction required to achieve discontinuous shear thickening may coincide with an underlying phase transition in the dispersion. Several systems in the literature illustrate orientationally-ordered phases, such as rod-like colloidal suspensions of tobacco mosaic virus, boehmite, and cellulose microcrystals [Langmuir (1938); Buining et al. (1994); Zocher and Torok (1962); Hermans (1963)]. This isotropic-nematic transition results when the excluded volume contribution to entropy overrides the loss of particle orientational entropy above some critical particle volume fraction [Onsager (1949)]. Below some critical aspect ratio $(L / D \sim 2.75)$, fluids of prolate ellipsoidal particles form a glassy phase prior to reaching the isotropic-nematic phase transition. The computer simulations of hard ellipsoid fluids of Lee (1988) predict isotropic-nematic phase transitions for prolate ellipsoidal particles with aspect ratios of 2.75 and 3, and simulations by Camp et al. (1996) have determined isotropic-nematic $(I-N)$ phase transitions at higher ellipsoidal aspect ratios $(L / D$ $=5-20)$. The solid and dashed lines in Fig. 7 represent the $I-N$ phase boundaries generated from the simulation results of both Lee (1988) and Camp et al. (1996). Interestingly, critical volume fractions for discontinuous shear thickening of the PCC(4)/PEG and PCC(7)/PEG suspensions coincide, to within experimental accuracy, with the simulation results for isotropic-nematic phase transition. However, there is no mechanistic understanding of this observation for spherical or prolate ellipsoid particle suspensions and so, the coincidence remains unexplained, but nonetheless, intriguing.

We observe flow alignment in all of the suspensions (additional Rheo-SANS measurements not presented here) and do not have clear evidence for an isotropic-nematic phase transition in the suspensions at rest. Because the average particle aspect ratio of the 
$\mathrm{PCC}(2) / \mathrm{PEG}$ dispersion is below the predicted minimum particle aspect ratio, no nematic phase transition should be possible in this dispersion. The polydispersity of the PCC(4)/ PEG and PCC(7)/PEG suspensions will broaden the isotropic-nematic phase transition, which may explain the lack of a sharp transition in our measured rheology. Theoretical and experimental studies of even binary rigid rod mixtures indicate that several phases may coexist in addition to the $I-N$ coexistence state, including isotropic-nematic-nematic, isotropic-isotropic, and nematic-nematic states [Abe and Flory (1978); Lekkerkerker et al. (1984); Varga et al. (2003)]. It must also be emphasized that most of our rheological measurements and SANS investigations have been limited by sample properties and instrumentation to relatively large rotational Peclet numbers $\left(\propto \dot{\gamma} \cdot D_{r 0}^{-1}\right.$, where $D_{r 0}$ values based on the average particle dimensions are listed in Table I), such that significant shear-induced alignment is expected and observed.

\section{CONCLUSIONS}

Reversible shear thickening is observed in model colloidal suspensions of acicular particles. Many of the rheological traits associated with the continuous and discontinuous shear thickening samples are similar to those observed in hard sphere colloidal suspensions, such as reversibility, fast transients, and discontinuous shear thickening at high particle concentrations with associated flow jamming and slip. An important difference is that the particle volume fraction required to reach discontinuous shear thickening decreases with increasing particle aspect ratio. Interestingly, the critical stress for the onset of shear thickening does not depend on particle aspect ratio, but rather follows predictions and data for hard sphere suspensions based on the minor axis dimension. Flow and Rheo-SANS measurements demonstrate the samples are flow aligning and that increasing particle concentration leads to higher degrees of alignment. Discontinuous shear thickening samples maintain a high degree of flow alignment in the shear thickened state, while continuous shear thickening samples show a weak reduction in alignment with increasing shear stresses in this regime.

The experimental evidence, including: (a) lack of significant flow hysteresis, (b) dependence of the critical stress for shear thickening on the minor axis but not the particle aspect ratio, (c) decreasing volume fraction required for discontinuous shear thickening with particle aspect ratio, (d) continuity in particle alignment in the discontinuous shear thickened state but weak misalignment in the continuous shear thickened state, and (e) the quantitative agreement for the critical stress required for shear thickening with hard spherical particle suspensions, provides substantial evidence that the mechanism for shear thickening in these acicular particle suspensions is a consequence of hydrocluster formation arising from short-range hydrodynamic interactions. Previous hypotheses suggesting particle misalignment and "entanglement" as the mechanism responsible for the observed discontinuous shear thickening transition during simple shearing flows are shown to be invalid. This is most evident from Rheo-SANS experiments performed on the discontinuously shear thickening PCC(7)/PEG dispersion $(\phi=0.35)$, which demonstrate that the high degree of particle alignment that develops near the critical stress is maintained during discontinuous shear thickening, as well as the transient rheology performed on the PCC(7)/PEG sample just below the critical volume fraction $(\phi=0.30)$. This work motivates a need for a quantitative theory for the rheology and microstructure of suspensions of acicular particles that properly includes the short-range hydrodynamic interactions, which are observed to dominate the shear thickening regime. 


\section{ACKNOWLEDGMENTS}

This work has been supported by IFPRI and the Army Research Laboratory CMT program (Grant No. 33-21-3144-69) through the Center for Composite Materials of the University Delaware. R.G.E. acknowledges support from the NASA Delaware Space Grant College and Fellowship Program (NASA Grant No. NGT5-40024). The authors are grateful to Professor Lennart Bergstrom (Institute for Surface Chemistry, Stockholm, Sweden) for useful discussions, Dr. Lionel Porcar for assistance with the SANS measurements, Dr. Eric Wetzel (Army Research Laboratory) for research support, and to Dr. Douglas Donigian (Specialty Minerals, Inc.) for providing the aqueous PCC suspensions used in this investigation. The authors acknowledge the support of the National Institute of Standards and Technology, U.S. Department of Commerce, in providing the neutron research facilities used in this work. This work utilized facilities supported in part by the National Science Foundation under Agreement No. DMR-9986442.

\section{References}

Abe, A., and P. J. Flory, "Statistical thermodynamics of mixtures of rodlike particles. 2. Ternary systems," Macromolecules 11, 1122-1126 (1978).

Alince, B., and P. Lepoutre, "Flow behavior of pigment blends," Tappi J. 66, 57-60 (1983).

Beazley, K. M., "Industrial aqueous suspensions," in Rheometry: Industrial Applications, edited by K. Walters (Research Studies Press, Chichester, 1980), pp. 339-413.

Bender, J., and N. J. Wagner, "Reversible shear thickening in monodisperse and bidisperse colloidal dispersions," J. Rheol. 40, 899-916 (1996).

Bender, J. W., and N. J. Wagner, "Optical measurement of the contributions of colloidal forces to the rheology of concentrated suspensions," J. Colloid Interface Sci. 172, 171-185 (1995).

Bergstrom, L., "Rheological properties of $\mathrm{Al}_{2} \mathrm{O}_{3}-\mathrm{SiC}$ whisker composite suspensions," J. Mater. Sci. 31, 5257-5270 (1996).

Bergstrom, L., "Shear thinning and shear thickening of concentrated ceramic suspensions," Colloids Surf., A 133, 151-155 (1998).

Boersma, W. H., J. Laven, and H. N. Stein, "Viscoelastic properties of concentrated shear-thickening dispersions," J. Chem. Phys. 149, 10-22 (1992).

Brady, J. F., and G. Bossis, "Stokesian dynamics," Annu. Rev. Fluid Mech. 20, 111-157 (1988).

Buining, P. A., A. P. Philipse, and H. N. W. Lekkerkerker, "Phase behavior of aqueous dispersions of colloidal boehmite rods," Langmuir 10, 2106-2114 (1994).

Camerel, F., J. C. P. Gabriel, P. Batail, P. Panine, and P. Davidson, "Combined SAXS-rheological studies of liquid crystalline dispersions of mineral particles," Langmuir 19, 10028-10035 (2003).

Camp, P. J., C. P. Mason, M. P. Allen, A. A. Khare, and D. A. Kofke, "The isotropic-nematic phase transition in uniaxial hard ellipsoid fluids: Coexistence data and the approach to the Onsager limit," J. Chem. Phys. 105, 2837-2849 (1996).

Catherall, A. A., J. R. Melrose, and R. C. Ball, "Shear thickening and order-disorder effects in concentrated colloids at high shear rates," J. Rheol. 44, 1-25 (2000).

Chow, M. K., and C. F. Zukoski, "Gap size and shear history dependencies in shear thickening of a suspension ordered at rest," J. Rheol. 39, 15-32 (1995).

Clarke, B., "Rheology of coarse settling suspensions," Trans. Inst. Chem. Eng. 45, 251-252 (1967).

Clarke, S. M., A. R. Rennie, and P. Convert, "A diffraction technique to investigate the orientational alignment of anisotropic particles: studies of clay under flow," Europhys. Lett. 35, 233-238 (1996).

Dellimore, J. W., and R. G. Gosling, "Use of electrical conductance measurements in studies of orientation of microscopic particles in stationary and flowing suspensions," J. Appl. Phys. 44, 5599-5606 (1973).

D'Haene, P., J. Mewis, and G. G. Fuller, "Scattering Dichroism measurements of flow-induced structure of a shear thickening suspension," J. Colloid Interface Sci. 156, 350-358 (1992). 
Gopalakrishnan, V., and C. F. Zukoski, "Effect of attractions on shear thickening in dense suspensions," J. Rheol. 48, 1321-1344 (2004).

Henson, D. J., and M. E. Mackay, "Effect of gap on the viscosity of monodisperse polystytrene melts: Slip effects," J. Rheol. 39, 359-373 (1994).

Hermans, J., "Flow of gels of cellulose microcrystals. I. Random and liquid crystalline gels," J. Polym. Sci., Part C: Polym. Symp. 2, 129 (1963).

Hoffman, R. L., "Discontinuous and dilatant viscosity behavior in concentrated suspensions. I. Observation of a flow instability," Trans. Soc. Rheol. 16, 155-173 (1972).

Hoffman, R. L., "Discontinuous and dilatant viscosity behavior in concentrated suspensions II. Theory and experimental tests," J. Colloid Interface Sci. 46, 491-506 (1974).

Jogun, S. M., and C. F. Zukoski, "Rheology of dense suspensions of platelike particles,” J. Rheol. 40, 12111232 (1996).

Kaffashi, B., V. T. O’Brien, M. E. Mackay, and S. M. Underwood, "Elastic-like and viscous-like components of the shear viscosity for nearly hard sphere, Brownian suspensions," J. Colloid Interface Sci. 187, 22-28 (1997).

Kernick, W. A., and N. J. Wagner, "The role of liquid-crystalline polymer rheology on the evolving morphology of immiscible blends containing liquid-crystalline polymers," J. Rheol. 43, 521-549 (1999).

Langmuir, I., "The role of attractive and repulsive forces in the formation of tactoids, thixotropic gels, protein crystals and coacervates," J. Chem. Phys. 6, 873-896 (1938).

Laun, H. M., "Rheological properties of aqueous polymer dispersions," Angew. Makromol. Chem. 123, 335359 (1984).

Laun, H. M., R. Bung, S. Hess, W. Loose, O. Hess, K. Hahn, E. Hadicke, R. Hingmann, F. Schmidt, and P. Lindner, "Rheological and small angle neutron scattering investigation of shear-induced particle structures of concentrated polymer dispersions submitted to plane Poiseulle and Couette flow," J. Rheol. 36, 743-787 (1992).

Lee, D. I., and A. S. Reder, "The rheological properties of clay suspensions, latexes and clay-latex systems," in Proceedings of the 1972 TAPPI Coating Conference, San Francisco, 1972, pp. 201-231.

Lee, S.-D., "The Onsager-type theory for nematic ordering of finite-length hard ellipsoids," J. Chem. Phys. 89, $7036-7037$ (1988).

Lekkerkerker, H. N. W., P. Coulon, and R. Van Der Haegen, "On the isotropic-liquid crystal phase separation in a solution of rodlike particles of different lengths," J. Chem. Phys. 80, 3427-3433 (1984).

Maranzano, B. J., "Rheology and microstructure of concentrated near hard sphere colloidal dispersions at the shear thickening transition," Ph.D. thesis, University of Delaware, 2001.

Maranzano, B. J., and N. J. Wagner, "The effects of particle size on reversible shear thickening of concentrated colloidal dispersions," J. Chem. Phys. 114, 10514-10527 (2001a).

Maranzano, B. J., and N. J. Wagner, "The effects of interparticle interactions and particle size on reversible shear thickening: Hard-sphere colloidal dispersions," J. Rheol. 45, 1205 (2001b).

Maranzano, B. J., and N. J. Wagner, "Flow-small angle neutron scattering measurements of colloidal dispersion microstructure evolution through the shear thickening transition," J. Chem. Phys. 117, 10291-10302 (2002).

Newstein, M. C., H. Wang, N. P. Balsara, A. A. Lefebvre, Y. Shnidman, H. Watanabe, K. Osaki, T. Shikata, H. Niwa, and Y. Morishima, "Microstructural changes in a colloidal liquid in the shear thinning and shear thickening regimes," J. Chem. Phys. 111, 4827-4838 (1999).

O'Brien, V. T., and M. E. Mackay, "Stress components and shear thickening of concentrated hard sphere suspensions," Langmuir 16, 7931-7938 (2000).

O’Brien, V. T., and M. E. Mackay, "Shear and elongational flow properties of kaolin suspensions," J. Rheol. 46, 557-572 (2002).

Okubo, T., "Rotational Brownian movement of tungstic acid colloids as studied by the spectrophotometric and conductance stopped-flow techniques," J. Am. Chem. Soc. 109, 1913-1916 (1987).

Onsager, L., "The effects of shape on the interaction of colloidal particles," Ann. N.Y. Acad. Sci. 51, 627-659 (1949).

Phan, S. E., W. B. Russel, Z. Cheng, J. Zhu, P. M. Chaikin, J. H. Dunsmuir, and R. H. Ottewill, "Phase 
transition, equation of state, and limiting shear viscosities of hard sphere dispersions," Phys. Rev. E 54, 6633-6645 (1996).

Philipse, A. P., "The random contact equation and its implications for (colloidal) rods in packings, suspensions, and anisotropic powders," Langmuir 12, 1127-1133 (1996).

Pusey, P. N., and W. van Megen, "Observation of a glass transition in suspensions of spherical colloidal particles," Phys. Rev. Lett. 59, 2083-2086 (1987).

Ramsay, J. D. F., and P. Linder, "Small-angle neutron scattering investigations of the structure of thixotropic dispersions of smectite clay colloids," J. Chem. Soc., Faraday Trans. 89, 4207-4214 (1993).

Varga, S., A. Galindo, and G. Jackson, "New types of phase behaviour in binary mixtures of hard rod-like particles," Mol. Phys. 101, 817-825 (2003).

Walker, L. M., and N. J. Wagner, "SANS analysis of the molecular order in poly( $\gamma$-benzyl L-glutamate)/ deuterated dimethylformamide (PBLG/d-DMF) under shear and during relaxation," Macromolecules 29, 2298-2301 (1996).

Yoshimura, A., and R. K. Prud'homme, "Wall slip corrections for Couette and parallel disk viscometers," J. Rheol. 32, 53-67 (1988).

Zocher, H., and C. Torok, "Tactosols. III. Aluminum hydroxide tactosols with diagonal structures," Kolloid Z. Z. Polym. 180, 41-51 (1962). 